\title{
Molecule-based coherent light-spin interfaces for quantum information processing - optical spin state polarization in a binuclear Europium complex
}

Kuppusamy Senthil Kumar

Karlsruhe Institute of Technology https://orcid.org/0000-0002-1501-7759

Diana Serrano

Chimie ParisTech

Aline Nonat

Laboratoire d'Ingénierie Moléculaire Appliquée à l'Analyse

Benoît Heinrich

University of Strasbourg

Lydia Karmazin

University of Strasbourg https://orcid.org/0000-0003-0416-6190

Loïc Charbonnière

CNRS / Université de Strasbourg

Philippe Goldner

Chimie ParisTech https://orcid.org/0000-0001-8517-0911

Mario Ruben ( $\nabla$ mario.ruben@kit.edu )

KIT-Karlsruhe https://orcid.org/0000-0002-7718-7016

\section{Article}

Keywords: quantum information processing, rare-earth ion-based molecular systems, polarization

Posted Date: January 7th, 2021

DOl: https://doi.org/10.21203/rs.3.rs-131741/v1

License: (1) (1) This work is licensed under a Creative Commons Attribution 4.0 International License. Read Full License

Version of Record: A version of this preprint was published at Nature Communications on April 12th, 2021. See the published version at https://doi.org/10.1038/s41467-021-22383-x. 


\section{Molecule-based coherent light-spin interfaces for quantum}

\section{information processing - optical spin-state polarization in a}

\section{binuclear europium complex}

Kuppusamy Senthil Kumar, ${ }^{1,2 *+}$ Diana Serrano, ${ }^{3 *+}$ Aline M. Nonat, ${ }^{4}$ Benoît Heinrich, ${ }^{1}$ Lydia

Karmazin, ${ }^{5}$ Loïc J. Charbonnière, ${ }^{4}$ Philippe Goldner, ${ }^{3 *}$ and Mario Ruben ${ }^{2,6,7 *}$

${ }^{1}$ Institut de Physique et Chimie des Matériaux de Strasbourg (IPCMS), CNRS-Université de Strasbourg, 23, rue du Loess, BP 43, 67034 Strasbourg cedex 2, France.

${ }^{2}$ Institute of Nanotechnology, Karlsruhe Institute of Technology (KIT), Hermann-von-Helmholtz-Platz 1, 76344, Eggenstein-Leopoldshafen, Germany.

${ }^{3}$ Institut de Recherche de Chimie Paris (IRCP), Université PSL, Chimie ParisTech, CNRS, 75005 Paris, France.

${ }^{4}$ Equipe de Synthèse pour l'Analyse, IPHC, UMR 7178, CNRS-Université de Strasbourg, ECPM, 25 rue Becquerel, 67087 Strasbourg Cedex, France.

${ }^{5}$ Service de Radiocristallographie, Fédération de Chimie Le Bel FR2010 CNRS-Université de Strasbourg, 1 rue Blaise Pascal, BP 296/R8, 67008 Strasbourg cedex, France.

${ }^{6}$ Institute for Quantum Materials and Technologies (IQMT), Karlsruhe Institute of Technology (KIT), Hermannvon-Helmholtz-Platz 1, 76344, Eggenstein-Leopoldshafen, Germany.

7Université de Strasbourg (Unistra), Institute de Science et d’Ingénierie Supramoléculaire (ISIS), 8, Allée Gaspard Monge, F-67000 Strasbourg, France.

†These two authors have equally contributed.

*e-mail: senthil.kuppusamy2@kit.edu; diana.serrano@chimieparistech.psl.eu;

philippe.goldner@chimieparistech.psl.eu; mario.ruben@kit.edu

\section{Abstract}

The success of the emerging field of solid-state optical quantum information processing (QIP) critically depends on the access to resonant optical materials. Rare-earth ion (REI)based molecular systems, whose quantum properties could be tuned taking advantage of molecular engineering strategies, are one of the systems actively pursued for the implementation of QIP schemes. Herein, we demonstrate the efficient polarization of 
ground-state nuclear spins-a fundamental requirement for all-optical spin initialization and addressing - in a binuclear Eu(III) complex, featuring inhomogeneously broadened ${ }^{5} \mathrm{D}_{0} \rightarrow^{7} \mathrm{~F}_{0}$ optical transition. At $1.4 \mathrm{~K}$, long-lived spectral holes have been burnt in the transition: homogeneous linewidth $\left(\Gamma_{h}\right)=22 \pm 1 \mathrm{MHz}$, which translates as optical coherence lifetime $\left(T_{2 o p t}\right)=14.5 \pm 0.7 \mathrm{~ns}$, and ground-state spin population lifetime $\left(T_{1 \text { spin }}\right)=1.6 \pm 0.4 \mathrm{~s}$ have been obtained. The results presented in this study are a progressive step towards the realization of molecule-based coherent light-spin QIP interfaces.

\section{Introduction}

Quantum information processing (QIP) schemes, such as quantum-computing, -storage, and -communication, use the quantum nature of materials to process and manipulate information $^{1-4}$. In QIP, a dramatic improvement in computation time and secure data transmission can be achieved by creating superposition states with long coherence lifetimes $\left(T_{2}\right)^{5,6}$. Environmental fluctuations due to lattice phonons, molecular vibrations, and magnetic moments reduce the coherence lifetime of a superposition state ${ }^{7,8}$. Thus, to achieve superposition states with coherence lifetimes suitable for realistic applications, the coherent states must be placed in a non-fluctuating environment.

Optical qubit operations can be performed with systems featuring narrow linewidth optical transitions. Colour centers in diamond ${ }^{4}$, rare-earth ions (REIs) doped in host matrices ${ }^{2,9-13,}$ and luminescent organic dye molecules (dye impurity), featuring extremely narrow and stable luminescent lines ${ }^{14-17}$, are suitable systems to implement optical qubit operations. REI-doped systems are particularly well-suited for optical QIP applications due to the following intrinsic properties. REls feature long optical coherence lifetimes $\left(T_{2 o p t}\right)$, because the $4 \mathrm{f}-4 \mathrm{f}$ optical transitions, covering the whole visible and IR spectral range, are wellshielded from the surrounding environment by the outer $5 s$ and $6 p$ orbitals. The nuclear spin 
(I) containing REI-isotopes enable the creation of nuclear spin superposition states with long spin coherence lifetimes $\left(T_{2 \text { spin }}\right)$, useful for storing quantum states ${ }^{11,18,19}$. Importantly, the exceptionally good optical coherence lifetimes associated with the $4 \mathrm{f}-4 \mathrm{f}$ transitions allow for coherent optical addressing and manipulation of nuclear spin states ${ }^{20-22}$.

Non-Kramers REls with an even number of f-electrons-for example, Eu(III), $\operatorname{Pr}($ III), or Tm(III)-embedded in a matrix with low average magnetic moments have been extensively studied for the implementation of QIP schemes ${ }^{23}$. The ${ }^{5} \mathrm{D}_{0} \rightarrow{ }^{7} \mathrm{~F}_{0}$ transition of Eu(III) is of particular interest because the ${ }^{5} \mathrm{D}_{0} \rightarrow{ }^{7} \mathrm{~F}_{0}$ transition is an induced electric dipole transition ${ }^{24}$ and is largely unaffected by the magnetic field fluctuations arising from the surrounding environment; thereby, long optical coherence lifetimes are associated with the transition. The QIP utility of Eu(III)-doped ceramics, powders, crystals, and nanoparticles-featuring ${ }^{5} D_{0} \rightarrow{ }^{7} F_{0}$ transition - has been elucidated ${ }^{2,25-28}$. However, it is difficult to tailor-make such systems with desirable optical properties by means of chemical and physical manipulations, limiting the utility of such materials for QIP applications. On the other hand, the optical properties of molecular Eu(III) complexes can be easily tuned by ligand field and molecular energy level engineering approaches, as previously exploited for applications, such as temperature sensing and bioimaging ${ }^{29,30}$. Further, by synthesizing isotopically enriched nuclear spin-free ligands, minimization of phonon and nuclear spin mediated relaxation pathways could be obtained; thereby, high quantum efficiencies and long $T_{2 o p t}$ could be achieved. Importantly, Eu(III) complexes with isotopically pure emitting centers, for example, ${ }^{151} \mathrm{Eu}$ or ${ }^{153} \mathrm{Eu}$, both with $I=5 / 2$, can be synthesized via isotopologues coordination chemistry $^{31,32}$, enabling the use of nuclear spin states as storage states for QIP applications. Frequency domain techniques such as transient spectral hole burning (SHB) have been used to optically probe superposition state lifetimes in molecular ${ }^{33,34}$ and REI-doped systems, for 
example, $\mathrm{Eu}(\mathrm{III}): \mathrm{Y}_{2} \mathrm{O}_{3}{ }^{26}$. Transient spectral holes appear as dips in absorption or excitation spectrum when a portion of active ions do not show ground state absorption anymore after optical pumping and for a certain time duration. The utility of transient SHB for measuring coherence lifetimes relies on the following factors, (i) existence of inhomogeneously broadened transitions with $\Gamma_{\mathrm{inh}} \gg>\Gamma_{\mathrm{h}}$, where $\Gamma_{\mathrm{inh}}$ and $\Gamma_{\mathrm{h}}$ are the optical inhomogeneous and homogeneous linewidths, respectively; (ii) $\Gamma_{\mathrm{h}} \gg \Gamma_{\text {laser, }}$ where $\Gamma_{\text {laser }}$ is the excitation laser linewidth; and (iii) $\Gamma_{\mathrm{h}}<\tau^{-1}$ and/or $T_{1 \text { spin }}{ }^{-1}$, for SHB in the excited-state and/or in the groundstate nuclear spin levels, where $\tau$ and $T_{1 \text { spin }}$ are the optical excited-state lifetime and the ground-state spin population lifetime, respectively. In these conditions, the hole width $\left(\Gamma_{\text {hole }}\right)$ is equal to $2 \Gamma_{\mathrm{h}}$, with $\Gamma_{\mathrm{h}}$ inversely proportional to the optical coherence lifetime $\left(\Gamma_{\mathrm{h}}=\right.$ $\left.1 / \pi T_{2 o p t}\right)$.

Reports on SHB in REls are rather limited to REls dispersed in matrices, such as $\mathrm{Er}^{3+}: \mathrm{LiYF}_{4}$, $\mathrm{Nd}^{3+}: \mathrm{YVO}_{4}, \mathrm{Eu}^{3+}: \mathrm{Y}_{2} \mathrm{SiO}_{5}$, and $\mathrm{Eu}^{3+}: \mathrm{Y}_{2} \mathrm{O}_{3}{ }^{23}$. To the best of our knowledge, transient $\mathrm{SHB}$ in a molecular REI system-especially in an Eu(III) complex-is yet to be reported. In this study, we demonstrate-for the first time-transient SHB (see methods, section S2, and Fig. S1-S4 for experimental details) in the inhomogeneously broadened ${ }^{5} D_{0} \rightarrow{ }^{7} F_{0}$ optical transition of a binuclear molecular Eu(III) complex- $\left[\mathrm{Eu}_{2} \mathrm{Cl}_{6}(4-p i c N O)_{4}\left(\mu_{2}-4-p i c N O\right)_{2}\right] \cdot 2 \mathrm{H}_{2} \mathrm{O}\left(\left[\mathrm{Eu}_{2}\right]\right) ; 4$-picNO stands for 4-picoline-N-Oxide. The measured hole width yields a homogeneous linewidth of $22 \pm 1 \mathrm{MHz}$, which corresponds to $\mathrm{T}_{2 \text { opt }}$ of $14.5 \pm 0.7 \mathrm{~ns}$. A hole decay time of $1.6 \pm 0.4 \mathrm{~s}$ is observed, consistent with nuclear spin relaxation. This confirms the utility of the SHB

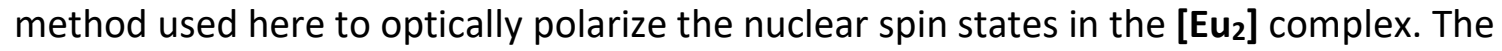
results presented in this study might open a new avenue in quantum materials research direction by placing the focus on REI-based molecular materials ${ }^{3,35}$. 


\section{Results}

\section{Design considerations, preparation, and X-ray structure analysis of [Eu $]$}

To implement optical QIP schemes, a molecular Eu(III)-complex should feature a long coherence time, photostability, stability to be handled at ambient conditions, and a reasonable luminescence quantum yield. Importantly, a low symmetry environment around the $\mathrm{Eu}(\mathrm{III})$ center should be satisfied to observe the ${ }^{5} \mathrm{D}_{0} \rightarrow^{7} \mathrm{~F}_{0}$ transition.

R-Pyridine-N-oxide (RPYNO; $\mathrm{R}=\mathrm{H}, \mathrm{CH}_{3}$, and $\mathrm{CF}_{3}$ ) ligands are suitable for the development of Eu(III)-complexes for QIP applications because such ligands feature nuclear spin-free oxygen donor sites and sensitize the Eu(III)-luminescence via antenna effect $24,36,37$. Moreover, the $\mu_{2^{-}}$ coordination mode of RPYNO ligands facilitates the formation of low-coordinate binuclear Eu-complexes with desired ligand field symmetry around the Eu(III) center, promoting the appearance of ${ }^{5} D_{0} \rightarrow{ }^{7} F_{0}$ transition. On the downside, RPYNO ligands feature nuclear spin containing hydrogen $\left({ }^{1} \mathrm{H}\right.$; abundance $>99.98 \%$ and $\left.\mathrm{I}=1 / 2\right)$ atoms in their backbone, which facilitate the decoherence of a superposition state. Therefore, the number of multiatomic ligands - containing hydrogen atoms - coordinating with Eu(III) should be reduced to improve the coherence time.

A reduced number of hydrogen-containing ligands around Eu(III) could be achieved employing monoatomic halide-based ligands-such as $\mathrm{Cl}$-as coordinating ligands in view of their heavier nature compared to hydrogen. On the other hand, $\mathrm{Cl}^{-}$ligands do not sensitize the $\mathrm{Eu}(\mathrm{III})$-luminescence and are composed of nuclear spin carrying isotopes $-{ }^{35} \mathrm{Cl}$ and ${ }^{37} \mathrm{Cl}$, both with I = 3/2. Remarkably, the gyromagnetic ratio $(\gamma)$ of nuclear spin isotopes of chlorine $-\mathrm{\gamma}=2.62 \times 10^{7} \mathrm{rad} \mathrm{s}^{-1} \mathrm{~T}^{-1}$ and $2.18 \times 10^{7} \mathrm{rad} \mathrm{s}^{-1} \mathrm{~T}^{-1}$ for ${ }^{35} \mathrm{Cl}$ and ${ }^{37} \mathrm{Cl}$, respectively-is about ten times smaller than the gyromagnetic ratio of hydrogen $\left(26.75 \times 10^{7} \mathrm{rad} \mathrm{s}^{-1} \mathrm{~T}^{-1}\right)$. 
Thus, the use of $\mathrm{Cl}^{-}$ligands is advantageous to reduce decoherence pathways arising from magnetic interactions.

Based on the above rationale, we have synthesized the binuclear $\left[\mathrm{Eu}_{2}\right]$ by taking advantage of the luminescence sensitization efficiency of a RPYNO ligand-4-picNO-and heavy and mono-atomic nature of $\mathrm{Cl}^{-}$ligand. Overall a trade-off between $\mathrm{Eu}(\mathrm{III})$ luminescence sensitization and decoherence reduction have been attempted.

The $\left[\mathrm{Eu}_{2}\right]$ complex was prepared by treating the commercially available $\mathrm{EuCl}_{3} \cdot 6 \mathrm{H}_{2} \mathrm{O}$ and 4picNO in 1:3 ratio in water followed by recrystallization of the crude reaction mixture from ethanol-ethyl acetate solvent mixture (see section S2.3 and scheme S1 for more details). The synthesis of the $\left[\mathrm{Eu}_{2}\right]$ complex is reproducible, and the method employed to prepare the complex was successfully extended to prepare a series of binuclear $\operatorname{Ln}(\mathrm{III})$ complexes$\left[\operatorname{Ln}_{2} \mathrm{Cl}_{6}(4-\text { picNO})_{4}\left(\mu_{2}-4-p i c N O\right)_{2}\right] \cdot 2 \mathrm{H}_{2} \mathrm{O}(\mathrm{Ln}=\mathrm{Tb}, \mathrm{Gd}, \mathrm{Dy}, \mathrm{Er}$, and Ho)-establishing the versatility of the procedure adopted. Since $\left[\mathrm{Gd}_{2} \mathrm{Cl}_{6}(4-\text { pic NO})_{4}\left(\mu_{2}-4-\text { picNO}\right)_{2}\right] \cdot 2 \mathrm{H}_{2} \mathrm{O}\left(\left[\mathrm{Gd}_{2}\right]\right)$ was used to estimate the triplet energy $\left({ }^{3} \pi \pi\right)$ of the 4-picNO ligand, the preparation and structural characterization of $\left[\mathbf{G d}_{\mathbf{2}}\right]$ is presented in the SI; see sections $\mathrm{S} 2.3$ and S3. X-ray crystal structures and the associated photophysical and magnetic properties of the remaining complexes $-(\mathrm{Ln}=\mathrm{Tb}, \mathrm{Dy}, \mathrm{Er}$, and $\mathrm{Ho})-$ will be reported elsewhere.

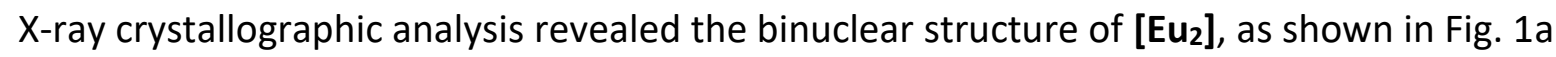
and b; see Fig. S5 and table S1 for packing in the crystal lattice and crystallographic data, respectively. The complex crystallized in the centrosymmetric $P \overline{1}$ space group, belonging to the triclinic crystal system. The neutral Eu(III)-dimer is composed of six 4-picNO ligands and six chloride ligands. The $\mathrm{O}_{4} \mathrm{Cl}_{3}$ coordination environment around each $\mathrm{Eu}(\mathrm{III})$ ion is best described as pentagonal bipyramidal with a continuous shape measure (CShM) ${ }^{38}$ of 1.513 , as shown in Fig. 1b. In [Eu $]$, the equatorial positions (edges) of each pentagonal bipyramid are 
occupied by two monodentate 4-picNO ligands in trans fashion, one chloride ligand, and two $\mu_{2}$-4-picNO ligands. The axial positions of each pentagonal bipyramid are occupied by the remaining chloride ligands. The intramolecular Eu $\cdots$ Eu distance is 4.273(4) $\AA$. A good match between the powder (PXRD) and single crystal (SCXRD) patterns (Fig. S6) and the similar unit cell parameters (Table S2) obtained from the indexing of the PXRD and SCXRD data unambiguously prove the phase purity of the crystalline material utilized for photophysical and SHB studies.

(a)

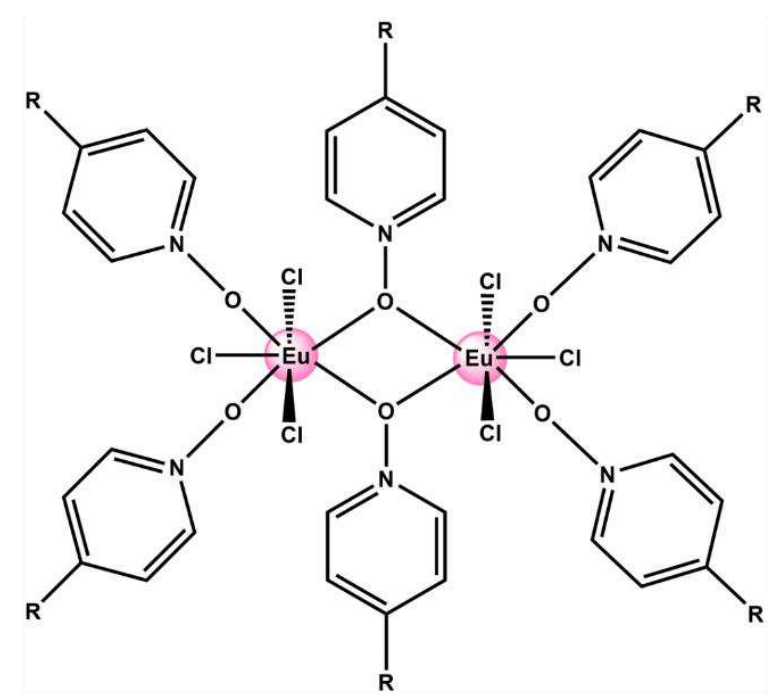

(b)

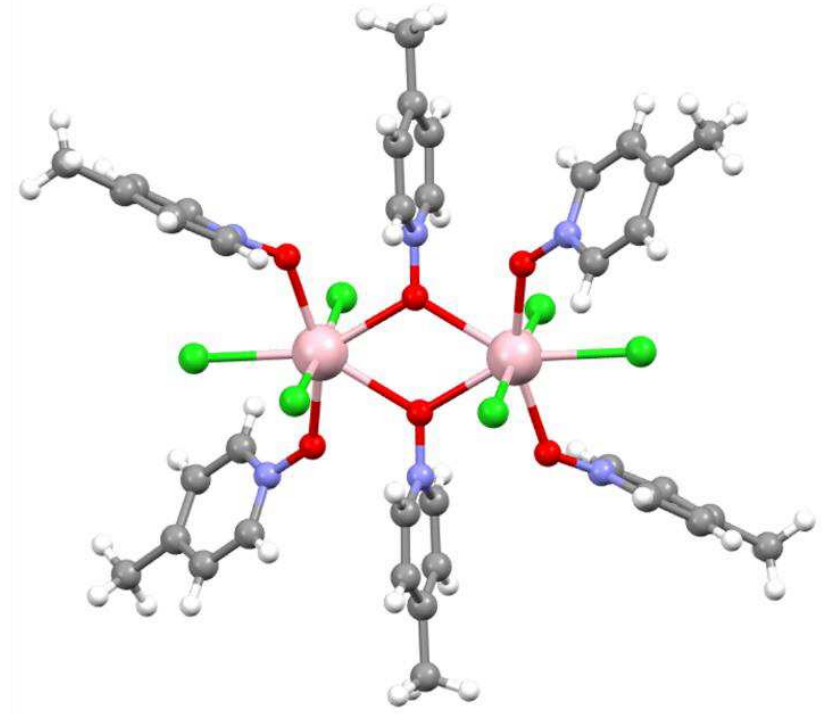

Fig. 1 | Structure of $\left[\mathrm{Eu}_{2} \mathrm{Cl}_{6}(4-p i c N O)_{4}\left(\mu_{2}-4-p i c N O\right)_{2}\right] \cdot 2 \mathrm{H}_{2} \mathrm{O}$. (a) Molecular structure of the complex showing the ligands $\left(\mathrm{R}=\mathrm{CH}_{3}\right)$ coordinating with the $\mathrm{Eu}(\mathrm{III})$ centers. The complex 
was prepared by treating $\mathrm{EuCl}_{3} \cdot 6 \mathrm{H}_{2} \mathrm{O}$ with 4-picNO ligand dissolved in water followed by a recrystallization step from ethanol (EtOH)/ethyl acetate (EtOAc) solvent mixture. (b) X-ray crystal structure of the complex. Coordination geometry around each Eu(III) center of the complex is best described as pentagonal bipyramidal. The co-crystallized water molecules are omitted for clarity. Colour code: $\mathrm{C}$, grey; $\mathrm{Cl}$, green; Eu, pink; $\mathrm{H}$, white; $\mathrm{N}$, blue; $\mathrm{O}$, red.

\section{Photophysical studies}

[Eu $\mathrm{Eu}_{2}$ exhibited sensitized (Fig. 2a) Eu(III)-based emission upon excitation of 4-picNO-based transition centered around $330 \mathrm{~nm}$. Among the ${ }^{5} D_{0} \rightarrow{ }^{7} F_{J}(J=0-6)$ transitions observed (Fig. $2 b$ ), the ${ }^{5} D_{0} \rightarrow{ }^{7} F_{0}$ transition is of interest to this study. The sharp and non-degenerate (vide infra) nature of the ${ }^{5} D_{0} \rightarrow{ }^{7} F_{0}$ transition is in agreement with identical environments around the two Eu(III) centers in the dimer. However, since the two sites have different orientations within the molecule, they are inequivalent and they can be differentiated, for example, upon application of an external magnetic field.

(a)

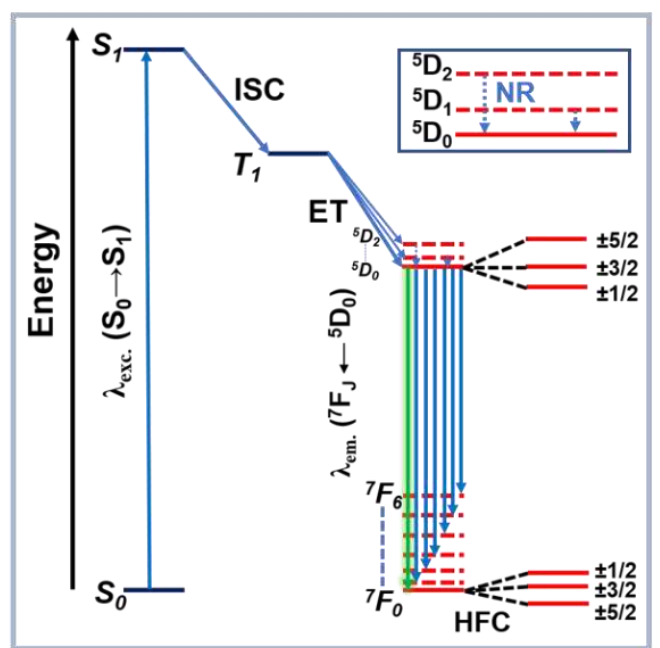

(c)

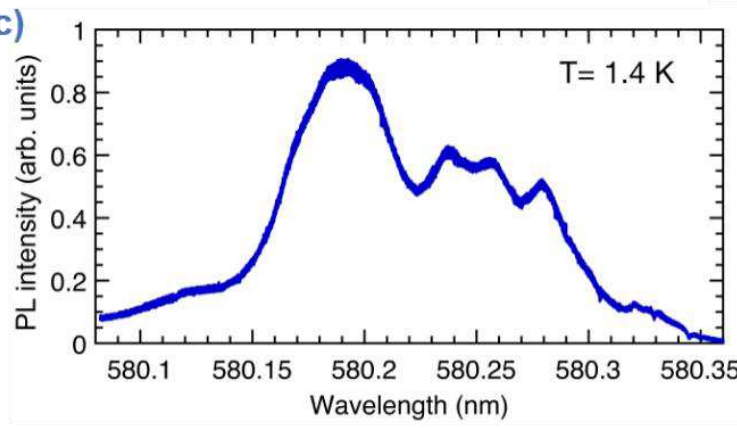

(b)

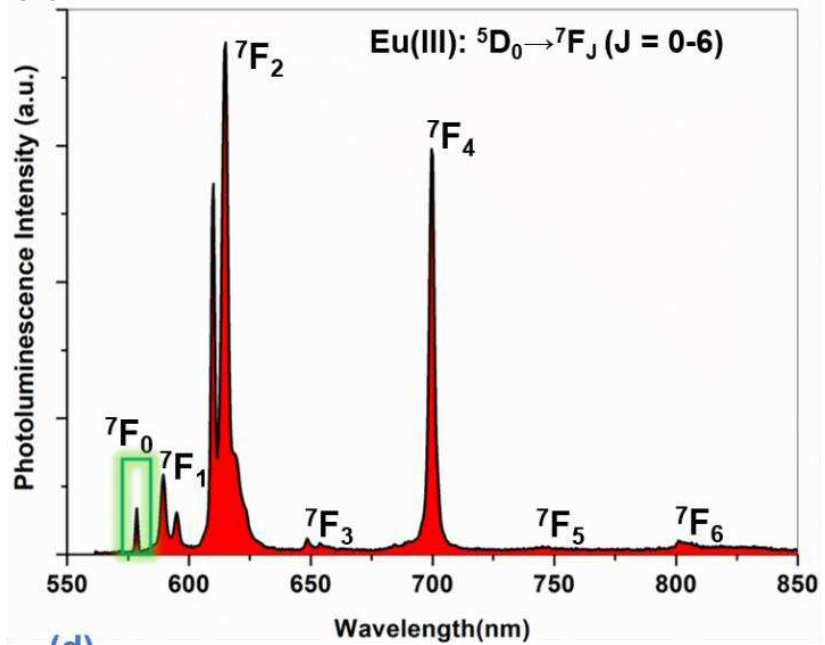

(d)

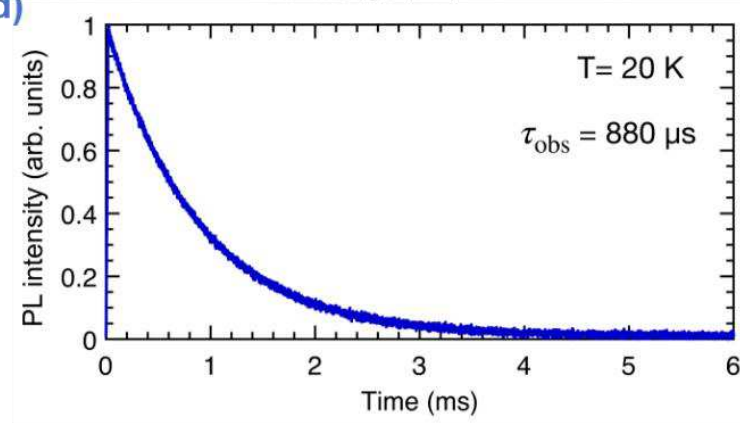




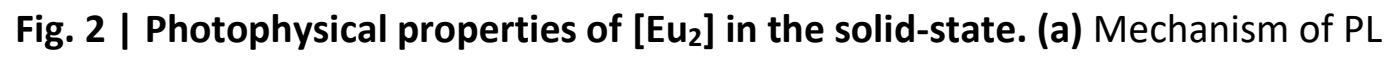

sensitization in $\mathrm{Eu}(\mathrm{III})$ complexes: The $\mathrm{Eu}(\mathrm{III})$-based ${ }^{5} \mathrm{D}_{J}(J=0-2)$ receiving levels are populated after a series of excitation, intersystem crossing (ISC), and $T_{1} \rightarrow^{5} D_{\text {j }}$ energy transfer (ET) processes. The co-populated excited ${ }^{5} D_{2}$ and ${ }^{5} D_{1}$ levels non-radiatively (NR) relax to ${ }^{5} D_{0}$ level (inset); radiative relaxation of ${ }^{5} D_{0}$ level to ground ${ }^{7} F_{J}(J=0-6)$ crystal field levels manifests as line-like luminescence. The ${ }^{5} D_{0} \rightarrow{ }^{7} F_{0}$ transition is suited for QIP applications due to its narrow linewidth and long coherence lifetimes of the nuclear spin states $- \pm 5 / 2, \pm 3 / 2, \pm 1 / 2(I=5 / 2$ for ${ }^{151} \mathrm{Eu} /{ }^{153} \mathrm{Eu}$ )-associated with the ${ }^{7} \mathrm{~F}_{0}$ ground-state level. (b) Photoluminescence spectrum showing the ${ }^{5} D_{0} \rightarrow{ }^{7} F_{J}(J=0-6)$ transitions in the visible and near-IR range $\left(\lambda_{\text {exc. }}=330\right.$ $\mathrm{nm}$ ). (c) Photoluminescence excitation (PLE) spectrum of the ${ }^{5} \mathrm{D}_{0} \rightarrow^{7} \mathrm{~F}_{0}$ transition of [Eu $]$ measured at $1.4 \mathrm{~K}$. An inhomogeneous linewidth $\left(\Gamma_{\mathrm{inh}}\right)$ of $50 \mathrm{GHz}$ is calculated for the main peak centered at $580.185 \mathrm{~nm}$ (vac.) (d) Luminescence decay of the ${ }^{5} \mathrm{D}_{0}$ excited-state of [Eu $\mathbf{E}_{2}$ ] measured at $20 \mathrm{~K}$ under resonant excitation at $580.185 \mathrm{~nm}$. An excited-state lifetime ( $\left.\tau_{\text {obs }}\right)$ of $880 \mu \mathrm{s}$ was obtained from a single exponential fit of the decay.

At RT, luminescence lifetime ( $\left.\tau_{o b s}\right)$ of $822 \mu \mathrm{s}$ (Fig. S1Ob) and total emission quantum yield $\left(Q_{t o t}\right)$ of $38 \pm 6 \%$ were determined for $\left[\mathrm{Eu}_{2}\right]$. See section S3.3.1 for a detailed analysis of the

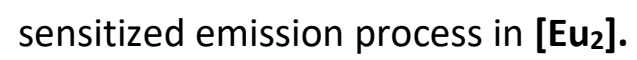

\section{Low-temperature high-resolution spectroscopy and spectral hole burning}

The ${ }^{5} D_{0} \rightarrow{ }^{7} F_{0}$ transition of Eu(III) is of particular interest for QIP and optical quantum technologies. The photoluminescence excitation (PLE) spectrum, recorded at $1.4 \mathrm{~K}$, of the ${ }^{5} \mathrm{D}_{0} \rightarrow{ }^{7} \mathrm{~F}_{0}$ optical transition in [Eu $\mathrm{Eu}_{2}$ (Fig. $2 \mathrm{c}$ ) is composed of several partially resolved peaks. The most prominent appeared at $580.185 \mathrm{~nm}$ (vac.) with a full width at half maximum 
(FWHM) of $0.06 \mathrm{~nm}\left(50 \mathrm{GHz}\right.$ or $\left.1.7 \mathrm{~cm}^{-1}\right)$. This is comparable to inhomogeneous linewidths observed in $\mathrm{Cr}(\mathrm{III})$ compounds $^{39}$, although larger than optical inhomogeneous linewidths observed in most inorganic crystals, in the few $\mathrm{GHz}$ range ${ }^{40}$. A $\tau_{\text {obs }}=880 \mu$ s was obtained from a single exponential fit of the ${ }^{5} \mathrm{D}_{0}$ fluorescence decay measured at $20 \mathrm{~K}$ under resonant excitation at $580.185 \mathrm{~nm}$ (Fig. 2d).

The complexity of this absorption spectrum for a transition taking place between two singlet electronic levels, and Eu(III) ions placed in an identical ligand field environment in each [Eu $\mathbf{E}_{2}$ ], raises the question of the origin of the different peaks. Selective excitation of the different peaks (Fig. 2c) reveals differences in the low-temperature PL emission spectra and observed lifetimes (Fig. S12). This indicates the existence of sub-site structures ${ }^{41,42}$ along with main

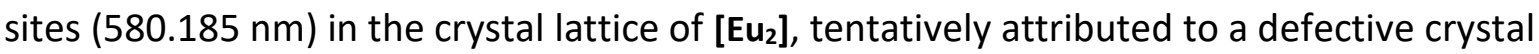
lattice. The single exponential character of the decay profiles, obtained after exciting the different peaks in Fig 2c, reveals the absence of interactions between Eu(III) centers in different sub-sites.

Homogeneous linewidths are of major importance to evaluate the potential of a system for optical QIP applications. In particular, narrow homogeneous linewidths are required for optical quantum storage and subsequent quantum coherence transfer to the nuclear spin states. Homogeneous linewidth values are, however, difficult to access from the inhomogeneously broadened lines of REI-based systems by classical absorption and fluorescence techniques. To circumvent this issue, we have used $\mathrm{SHB}^{43}$ and probed the

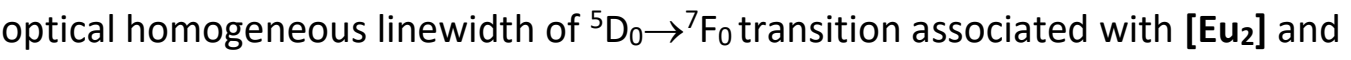
demonstrated selective optical addressing of the ground-state nuclear spin levels in the complex (see methods, section S2, and Fig. S2-S4 for details). The applied optical pumping continuously transfers a resonant ensemble of Eu(III) molecules to the excited state (Fig. 3b). 
Once in the excited-state, three decay channels are possible, because Eu(III) presents three doubly-degenerate ground-state nuclear spin levels $\left( \pm 5 / 2, \pm 3 / 2\right.$, and $\pm 1 / 2$; for ${ }^{151 / 153} E u, I=$ 5/2). Ions decaying back to the initial-state will be immediately pumped back to excitedstates; the ions relaxing to other nuclear spin states are off-resonant with the excitation laser; therefore, the population is stored in these states until spin relaxation occurs.
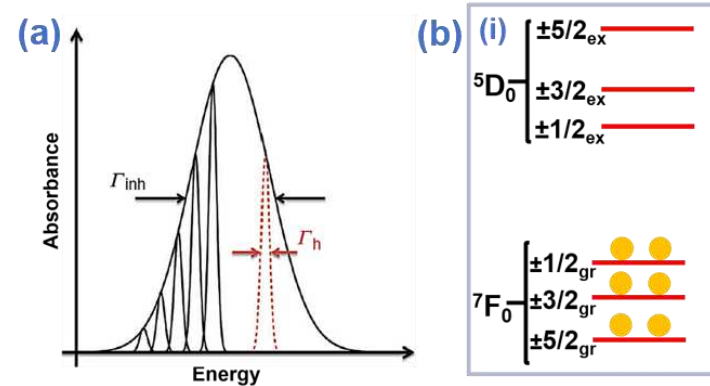

(c)

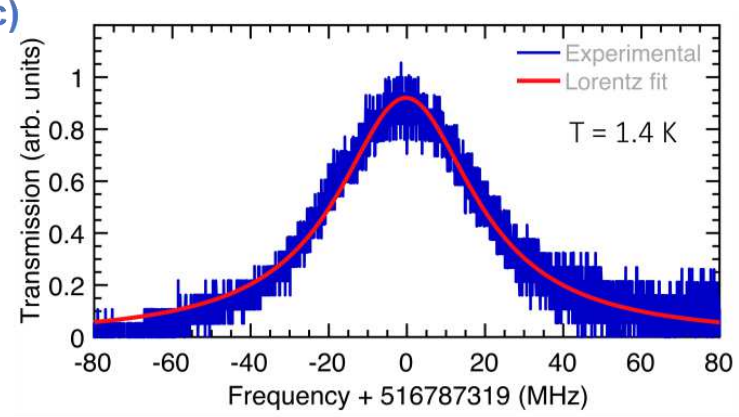

(d)

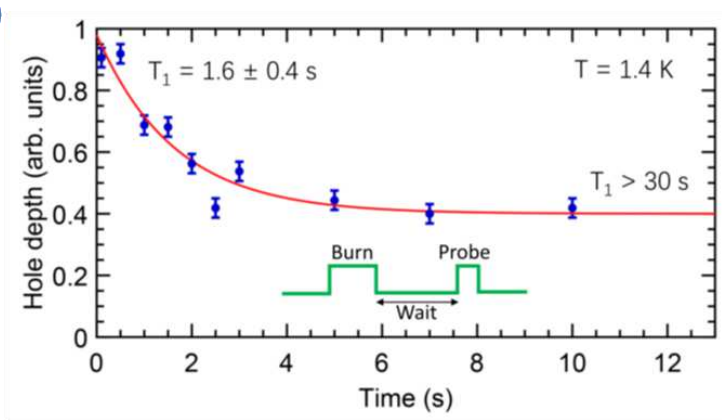

Fig. 3 | Spectral hole burning (SHB) in the ${ }^{5} \mathrm{D}_{0} \rightarrow^{7} \mathrm{~F}_{0}$ transition of [Eu $\left.\mathrm{Eu}_{2}\right]$ (a) An

inhomogeneously $\left(\Gamma_{\mathrm{inh}}\right)$ broadened line is composed of narrow homogeneously $\left(\Gamma_{\mathrm{h}}\right)$ broadened lines. Selective optical excitation of one or several homogeneously broadened lines is used to burn spectral holes with implications for QIP applications. (b) Simplified mechanism of SHB. In the diagram, $\pm 1 / 2_{\mathrm{gr}}, \pm 3 / 2_{\mathrm{gr}}, \pm 5 / 2_{\mathrm{gr}}$ and $\pm 1 / 2_{\mathrm{ex}}, \pm 3 / 2_{\mathrm{ex}}, \pm 5 / 2_{\mathrm{ex}}$ correspond to nuclear spin levels associated with the ground ${ }^{7} F_{0}$ and excited ${ }^{5} D_{0}$ states, respectively. (i) A laser scan reveals an inhomogeneously broadened absorption spectrum, like the one shown in (a), due to the excitation of multiple ions ensembles with infinitesimally small energy difference. (ii) Selective and continuous laser pumping transfers population from one of the ground-state spin levels to an excited-state spin level. This is 
illustrated for ions with the $\pm 5 / 2$ gr $\left({ }^{7} F_{0}\right)$ to $\pm 1 / 2_{\text {ex }}\left({ }^{5} D_{0}\right)$ transition resonant with the laser. (iii) Population relaxation from level $\pm 1 / 2$ ex to level $\pm 3 / 2 \mathrm{gr}$, under the ambit of level $\pm 3 / 2 \mathrm{gr}$ having sufficiently longer lifetime than levels $\pm 1 / 2 \mathrm{gr}$ and $\pm 5 / 2 \mathrm{gr}$, results in a decreased population in level $\pm 5 / 2$ gr. Since the spin level splittings are much lower than the optical inhomogeneous broadening, the laser will excite other ions along transitions from the $\pm 3 / 2 \mathrm{gr}$ or $\pm 1 / 2 \mathrm{gr}$ levels, which will therefore be emptied. This finally results in a hole in the inhomogeneously broadened absorption spectrum. (c) Spectral hole burned in the ${ }^{5} \mathrm{D}_{0} \rightarrow^{7} \mathrm{~F}_{0}$ transition of [Eu $\mathbf{E u}_{2}$. A Lorentzian fit reveals an FWHM of $43 \pm 2 \mathrm{MHz}$, corresponding to $\Gamma_{\mathrm{h}}=22 \pm 1 \mathrm{MHz}$. A $\mathrm{T}_{2 \mathrm{opt}}=$ $\sim 14.5 \mathrm{~ns}$ is calculated using the relation $\Gamma_{\mathrm{h}}=\left(1 / \pi \mathrm{T}_{2 \mathrm{opt}}\right)$. (d) The decay of the hole depth as a function of delay time before readout, showing the relaxation rate of the nuclear spin levels in the binuclear complex. Figures (a) and (b) are partially reproduced with permission from reference 23 .

As the pumping proceeds, the initial spin states are progressively emptied, and the population transferred to different spin levels (Fig. 3b). Scanning the laser over the optical inhomogeneous line then reveals an increased transmission - that is, a spectral hole-at the burning frequency. For an excitation laser linewidth much narrower compared to the measured hole widths $\left(\Gamma_{\text {hole }}\right), \Gamma_{\mathrm{h}}$ of a transition can be derived from the hole width $\left(\Gamma_{\mathrm{h}}=\Gamma_{\text {hole }} / 2\right)$. The SHB method is important as it is used for tailoring absorption profiles in several quantum storage protocols and spin population initialization ${ }^{21,44}$. A spectral hole burned in the ${ }^{5} \mathrm{D}_{0} \rightarrow{ }^{7} \mathrm{~F}_{0}$ transition of $\left[\mathrm{Eu}_{2}\right]$ is shown in Fig. 3c. The hole was fitted by a Lorentz peak function, yielding a hole width of $43 \pm 2 \mathrm{MHz}$ at FWHM, corresponding to $\Gamma_{\mathrm{h}}=22 \pm 1 \mathrm{MHz}\left(\mathrm{T}_{2 \mathrm{opt}}=14.5 \pm 0.7 \mathrm{~ns}\right)$. The observed $\Gamma_{\mathrm{h}}$ for $\left[\mathrm{Eu}_{2}\right]$ is relatively large compared to $\mathrm{Eu}(\mathrm{III})$ ions dispersed in matrices ${ }^{40}$. However, the $\Gamma_{\mathrm{h}}$ is about an 
order of magnitude lower than homogeneous linewidths observed for $\mathrm{Cr}$ (III) complexes diluted in amorphous host lattices ${ }^{39,45}$ and comparable to the ones reported for nitrogenvacancy (NV) centers in diamond ${ }^{46}$.

A lifetime limited homogeneous linewidth of $180 \mathrm{~Hz}$ was calculated for [ $\left.\mathrm{Eu}_{2}\right]$, using the experimentally observed excited state lifetime $\tau_{\mathrm{obs}}=880 \mu \mathrm{s}$ that includes local field correction effects $^{47,48}$. The homogeneous linewidth of $22 \pm 1 \mathrm{MHz}$ obtained from the SHB studies is therefore much larger than the lifetime limited homogeneous linewidth. We attribute this additional broadening to fluctuations in $\mathrm{Eu}(\mathrm{III})$ environment caused by molecular vibrations and rotations, as in $\mathrm{Cr}(\mathrm{III})$ complexes $^{45}$. Nuclear spin containing elements $(\mathrm{H}$ and $\mathrm{Cl})$ surrounding the $\mathrm{Eu}(\mathrm{III}) \operatorname{sites}^{49}$, as well as spectral diffusion arising due to disorder, defects, and grain boundaries ${ }^{45,50-52}$ could also have contributed to dephasing (see section S3.3.5). More insights on the dephasing processes could be obtained by performing temperature-dependent studies ${ }^{27,52}$, especially below $1 \mathrm{~K}$, where dephasing processes should be significantly reduced.

To further confirm that the observed spectral holes result from population transfer and storage in a different spin level, several criteria were verified. First, the determined minimum time delay $(5 \mathrm{~ms}$ ) between hole burning and hole readout pulses was much longer than the excited-state lifetime of $880 \mu \mathrm{s}$, ensuring that no population remained in the optical excited state (Fig. S3). Second, we confirmed that holes could be erased on demand by scanning the laser over a frequency of $200 \mathrm{MHz}$ with high intensity. These sweeping laser pulses excite all the spin population in the ground state, including the populations present in the storage levels (Fig. 3a), because typical Eu(III) ground state hyperfine splittings are in the range of $25-100 \mathrm{MHz}^{53}$. Thermal equilibrium population distribution is then reestablished following spontaneous relaxation from the excited state. Finally, we observed that holes 
could be erased and then burned again at a different frequency within the optical inhomogeneous line (Fig. S4), unambiguously elucidating selective and reversible optical addressing of a sub-ensemble of Eu(III) ions.

The decay of the hole depth was observed by increasing the delay between burning and readout pulses (Fig. $3 \mathrm{~d})$. This measurement provides insight into the relaxation times $\left(\mathrm{T}_{1 \text { spin }}\right)$ for the nuclear spin levels of Eu(III). Two components are observed in the experimental hole decay. A relaxation time of $1.6 \pm 0.4 \mathrm{~s}$ is obtained for the fast component by a single exponential fit. The second component is too slow to be accurately determined in the measurement time scale (10 s), but we estimate a lower bound relaxation time of $30 \mathrm{~s}$. Multiexponential hole decay curves have been previously observed in rare-earth materials ${ }^{27}$, due to different spin-lattice relaxation rates among the ground-state spin levels. For the fast component, $\mathrm{T}_{1 \text { spin }}$ is not limited by spin flip-flops between neighboring $\mathrm{Eu}(\mathrm{III})$ ions since the intramolecular flip-flop rate is expected between $10^{-4}$ and $10^{-5} \mathrm{~s}^{-154}$, which is remarkably lower than the relaxation rate $\left(\mathrm{T}_{1 \mathrm{spin}}{ }^{-1}\right)$ of $\sim 0.6 \mathrm{~s}^{-1}$ measured in the complex. Still, this relaxation time is more than three orders of magnitude larger than the excited-state lifetime; therefore, efficient spin population transfer can be achieved, enabling, for example, spin-state initialization.

\section{Discussion}

The foregoing experimental observations demonstrate the QIP utility of REI-based molecular complexes. On a comparative scale, the magnitude of optical coherence lifetime $\left(T_{2 o p t}\right)$ observed for $\left[\mathrm{Eu}_{2}\right]$ is several orders smaller than the values reported for Eu-doped systems. Unlike the doped systems, molecular systems offer the possibility to tune the optical properties via molecular engineering strategies. For example, ideal Eu(III) complexes showing long optical and spin coherence times could be obtained by designing ligands with 
deuterated backbones and nuclear spin-free donor sites and complexing such ligands with isotopically enriched REls. The molecular engineering strategy could also be adapted to obtain Eu(III) complexes with higher PL quantum yields than the $38 \pm 6 \%$ observed for [Eu 2$]$ (see the discussion in S3.3.1). On the flip side, designing of nuclear spin-free ligands or complete deuteration of structurally complex ligand backbones is a challenging task. Moreover, nuclear spins are difficult to address, rendering the qubit scalability based on molecular self-assembly a demanding task to achieve-synthesis of multinuclear REI systems is a strategy to overcome this issue ${ }^{31}$. In conclusion, long-lived spectral holes have been prepared in the inhomogeneously

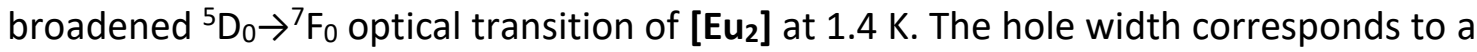
homogeneous linewidth $\left(\Gamma_{\mathrm{h}}\right)$ of $22 \pm 1 \mathrm{MHz}$, which is equivalent to an optical coherence lifetime $\left(T_{2 o p t}\right)$ of $14.5 \pm 0.7 \mathrm{~ns}$. The burn mechanism is attributed to population redistribution within the ground-state nuclear spin levels of Eu(III) that act as shelving levels. The observation of narrow optical inhomogeneous linewidth and nuclear spin state polarization associated with $\left[\mathrm{Eu}_{2}\right]$-reported for the first time in this study-relies on the presence of narrow ${ }^{5} \mathrm{D}_{0} \rightarrow^{7} \mathrm{~F}_{0}$ optical transition in the complex. The usefulness of such a transition for QIP applications could be enhanced by means of molecular engineering approaches. The tunable nature of molecular properties with atomic precision could provide access to quantum materials with technologically relevant spin and optical coherence lifetimes. Overall, the results presented in this study demonstrate the utility of REI-based molecular systems as coherent light-spin interfaces in optical QIP schemes. 


\section{Methods}

Experimental descriptions on spectral hole burning spectroscopy, preparation of the complex, X-ray crystallography, and powder X-ray diffraction analysis of the complex are detailed in the supporting information associated with this article.

\section{Steady-state photoluminescence spectroscopy}

Steady-state emission spectra were recorded on an FLP920 spectrometer from Edinburgh Instrument working with a continuous $450 \mathrm{~W}$ Xe lamp and a red-sensitive Hamamatsu R928 photomultiplier in Peltier housing. The emission spectrum of the europium complex in the NIR region was also measured by using a nitrogen-cooled Hamamatsu R5509-72 Vis-NIR (300 $1700 \mathrm{~nm}$ ), affording similar results. All spectra were corrected for the instrumental functions. When necessary, high pass filters at $330 \mathrm{~nm}, 395 \mathrm{~nm}, 455 \mathrm{~nm}$, or $850 \mathrm{~nm}$ were used to eliminate the second-order artifacts.

Fluorescence lifetimes were measured at room temperature on the same instrument working in the Multi Channels Spectroscopy (MCS) mode and using a Xenon flash lamp as the excitation source. Errors on lifetimes are $\pm 10 \%$. Luminescence quantum yields were measured with a G8 Integrating Sphere (GMP SA, Switzerland) according to the absolute method detailed in ref $^{55}$. Estimated errors are $\pm 15 \%$.

\section{Low-temperature high-resolution spectroscopy and spectral hole burning measurements}

Photoluminescence excitation (PLE), fluorescence lifetime, and spectral hole burning measurements were carried out on microcrystalline powder (Fig. S1). Five mg of total material was added into a home-built brass container with front and rear optical access (see Fig. S1). The filled container was then introduced into a He bath cryostat (Janis SVT-200). The sample temperature was regulated by acting on the liquid He volume and pressure in the sample chamber, and it was continuously monitored by a Si diode (Lakeshore DT-610). 
Optical excitation was carried out by a tunable CW dye laser (Sirah Matisse DS) with $250 \mathrm{kHz}$ linewidth. The excitation beam was focused onto the sample by a 75-mm-diameter lens and transmitted scattered light was collected by several lenses. Signals were detected with an avalanche photodiode (Thorlabs $110 \mathrm{~A} / \mathrm{M}$ ). The experimental setup is schematically represented in Fig. S1.

PLE spectra were recorded by scanning the excitation wavelength $\left(\lambda_{\text {exc. }}\right)$ between 580.08 and $580.35 \mathrm{~nm}$ (vac.) while monitoring the fluorescence intensity from the ${ }^{5} \mathrm{D}_{0}$ excited state to the ${ }^{7} F_{J}$ levels $(J=2-6)$. A long-pass filter (cut-off at $590 \mathrm{~nm}$ ) was placed in front of the detector to reject the excitation wavelength. Low-temperature emission spectra were recorded under different excitation wavelengths using a thermoelectrically cooled CCD spectrometer (Avantes, AvaSpec-2048). The crystalline material showed no evidence of photobleaching during low-temperature experiments, which was confirmed by monitoring fluorescence intensity under CW laser excitation (see Fig. S13).

SHB spectra and fluorescence decays were recorded by modulating the CW output of the laser in frequency and amplitude with an acousto-optic modulator (AOM; AA Optoelectronic MT200-B100A0, 5-VIS, $200 \mathrm{MHz}$ central frequency), set in the double-pass configuration, and driven by an arbitrary waveform generator with $625 \mathrm{MS} \mathrm{s}^{-1}$ sampling rate (Agilent N8242A). The ${ }^{5} D_{0}$ fluorescence decay was recorded after a single excitation pulse of $2 \mathrm{~ms}$ at 580.185 $\mathrm{nm}$. The SHB sequence was formed of ten burning pulses of $2 \mathrm{~ms}$ length, with an excitation power of $30 \mathrm{~mW}$ and at a fixed excitation wavelength within the inhomogeneous absorption profile. The waiting time between pulses was set to $5 \mathrm{~ms}$ to enable spontaneous relaxation from the excited state to the ground state nuclear spin levels before a new burn. The spectral hole was then probed by monitoring the transmission of a frequency scanning pulse of 2 ms duration, with a frequency span of $200 \mathrm{MHz}$ and excitation power of $5 \mathrm{~mW}$. The 
recorded transmission under burn conditions was corrected from transmission obtained without burning, to cancel out the frequency-dependent response of the acousto-optic modulator. The delay before readout was varied from a minimum of $5 \mathrm{~ms}$ to a maximum of 10 s. Every SHB sequence was ended by a series of high-power pulses scanning over 200 $\mathrm{MHz}$, to reset the ground-state population back to equilibrium. Spectral holes were recorded after averaging 50 sequences to improve the signal to noise ratio, for a better estimation of the hole width. The spectral width of the 2-millisecond-long burning pulse is here limited by the laser linewidth $(250 \mathrm{kHz})$, which is negligible compared to the measured spectral holes and also typical Eu(III) hyperfine splittings ${ }^{53}$. Hole linewidths were determined by Lorentz fit to the experimental data. Fluorescence lifetimes were determined by a single exponential fit to the decay data. The relaxation time for the fast component of the hole decay was also determined by a single exponential fit. The slow component lower bound limit was determined as the maximum decay possible in a time delay of $5 \mathrm{~s}$ within the experimental error bars given for the hole depths.

\section{References}

1. Hedges, M. P., Longdell, J. J., Li, Y. \& Sellars, M. J. Efficient quantum memory for light. Nature 465, $1052-1056$ (2010).

2. Kunkel, N. \& Goldner, P. Recent Advances in Rare Earth Doped Inorganic Crystalline Materials for Quantum Information Processing: Recent Advances in Rare Earth Doped Inorganic Crystalline Materials for Quantum Information Processing. Z. Für Anorg. Allg. Chem. 644, 66-76 (2018).

3. Moreno-Pineda, E., Godfrin, C., Balestro, F., Wernsdorfer, W. \& Ruben, M. Molecular spin qudits for quantum algorithms. Chem. Soc. Rev. 47, 501-513 (2018).

4. Awschalom, D. D., Hanson, R., Wrachtrup, J. \& Zhou, B. B. Quantum technologies with optically interfaced solid-state spins. Nat. Photonics 12, 516-527 (2018). 
5. Arute, F. et al. Quantum supremacy using a programmable superconducting processor. Nature 574, 505-510 (2019).

6. Korzh, B. et al. Provably secure and practical quantum key distribution over $307 \mathrm{~km}$ of optical fibre. Nat. Photonics 9, 163-168 (2015).

7. Eberly, J. \& Stroud Jr., C. Coherent Transients. in Springer Handbook of Atomic, Molecular, and Optical Physics (ed. Drake, G.) 1065-1076 (Springer New York, 2006). doi:10.1007/978-0-38726308-3_73.

8. Morgan, J. R., Chock, E. P., Hopewell, W. D., El-Sayed, M. A. \& Orbach, R. Origins of homogeneous and inhomogeneous line widths of the 5D0-7F0 transition of europium(3+) in amorphous solids. J. Phys. Chem. 85, 747-751 (1981).

9. Zhong, T. et al. Nanophotonic rare-earth quantum memory with optically controlled retrieval. Science 357, 1392-1395 (2017).

10. Siyushev, P. et al. Coherent properties of single rare-earth spin qubits. Nat. Commun. 5, (2014).

11. Zhong, M. et al. Optically addressable nuclear spins in a solid with a six-hour coherence time. Nature 517, 177-180 (2015).

12. Ortu, A. et al. Simultaneous coherence enhancement of optical and microwave transitions in solid-state electronic spins. Nat. Mater. 17, 671-675 (2018).

13. Phenicie, C. M. et al. Narrow Optical Line Widths in Erbium Implanted in $\mathrm{TiO}_{2}$. Nano Lett. 19, 8928-8933 (2019).

14. Ambrose, W. P. \& Moerner, W. E. Fluorescence spectroscopy and spectral diffusion of single impurity molecules in a crystal. Nature 349, 225-227 (1991).

15. Gorshelev, A. A. et al. Ortho-Dichlorobenzene Doped with Terrylene-a Highly Photo-Stable Single-Molecule System Promising for Photonics Applications. ChemPhysChem 11, 182-187 (2010).

16. Białkowska, M. et al. Single molecules of terrylene in di-substituted naphthalenes crystallizing in the herringbone pattern. RSC Adv. 7, 2780-2788 (2017). 
17. Navarro, P., Tian, Y., van Stee, M. \& Orrit, M. Stable Single-Molecule Lines of Terrylene in Polycrystalline para -Dichlorobenzene at 1.5 K. ChemPhysChem 15, 3032-3039 (2014).

18. Godfrin, C. et al. Operating Quantum States in Single Magnetic Molecules: Implementation of Grover's Quantum Algorithm. Phys. Rev. Lett. 119, (2017).

19. Thiele, S. et al. Electrically driven nuclear spin resonance in single-molecule magnets. Science 344, 1135-1138 (2014).

20. Raha, M. et al. Optical quantum nondemolition measurement of a single rare earth ion qubit. Nat. Commun. 11, (2020).

21. Kindem, J. M. et al. Control and single-shot readout of an ion embedded in a nanophotonic cavity. Nature (2020) doi:10.1038/s41586-020-2160-9.

22. Kornher, T. et al. Sensing Individual Nuclear Spins with a Single Rare-Earth Electron Spin. Phys. Rev. Lett. 124, (2020).

23. Goldner, P., Ferrier, A. \& Guillot-Noël, O. Rare Earth-Doped Crystals for Quantum Information Processing. in Handbook on the Physics and Chemistry of Rare Earths vol. 46 1-78 (Elsevier, 2015).

24. Binnemans, K. Interpretation of europium(III) spectra. Coord. Chem. Rev. 295, 1-45 (2015).

25. Serrano, D., Karlsson, J., Fossati, A., Ferrier, A. \& Goldner, P. All-optical control of long-lived nuclear spins in rare-earth doped nanoparticles. Nat. Commun. 9, (2018).

26. Bartholomew, J. G., de Oliveira Lima, K., Ferrier, A. \& Goldner, P. Optical Line Width Broadening Mechanisms at the $10 \mathrm{kHz}$ Level in $\mathrm{Eu}^{3+}: \mathrm{Y}_{2} \mathrm{O}_{3}$ Nanoparticles. Nano Lett. 17, 778-787 (2017).

27. Könz, F. et al. Temperature and concentration dependence of optical dephasing, spectral-hole lifetime, and anisotropic absorption in Eu $3+$ : Y 2 SiO 5. Phys. Rev. B 68, (2003).

28. Harada, N. et al. Chemically vapor deposited $\mathrm{Eu}^{3+}: \mathrm{Y}_{2} \mathrm{O}_{3}$ thin films as a material platform for quantum technologies. J. Appl. Phys. 128, 055304 (2020).

29. Brites, C. D. S. et al. A Luminescent Molecular Thermometer for Long-Term Absolute Temperature Measurements at the Nanoscale. Adv. Mater. 22, 4499-4504 (2010). 
30. Sy, M., Nonat, A., Hildebrandt, N. \& Charbonnière, L. J. Lanthanide-based luminescence biolabelling. Chem. Commun. 52, 5080-5095 (2016).

31. Wernsdorfer, W. \& Ruben, M. Synthetic Hilbert Space Engineering of Molecular Qu $d$ its: Isotopologue Chemistry. Adv. Mater. 31, 1806687 (2019).

32. Moreno-Pineda, E., Damjanović, M., Fuhr, O., Wernsdorfer, W. \& Ruben, M. Nuclear Spin Isomers: Engineering a Et ${ }_{4}$ N[DyPc 2 ] Spin Qudit. Angew. Chem. Int. Ed. 56, 9915-9919 (2017).

33. Naumov, A. V. Low-temperature spectroscopy of organic molecules in solid matrices: from the Shpol'skii effect to laser luminescent spectromicroscopy for all effectively emitting single molecules. Phys.-Uspekhi 56, 605-622 (2013).

34. Kozankiewicz, B. \& Orrit, M. Single-molecule photophysics, from cryogenic to ambient conditions. Chem Soc Rev 43, 1029-1043 (2014).

35. Gaita-Ariño, A., Luis, F., Hill, S. \& Coronado, E. Molecular spins for quantum computation. Nat. Chem. 11, 301-309 (2019).

36. Thompson, L. C. \& Kuo, S. C. Structure and absorption and fluorescence spectra of Eu(PyNO)8(C104)3. J. Common Met. 148, 173-179 (1989).

37. Butter, E. \& Seifert, W. Beziehungen zwischen Lumineszenzspektrum und Struktur von Seltenerdkomplexen. I. Quadratisch-antiprismatisch koordinierte Pyridin-N-Oxid-Komplexe des Europium(III). Z. Für Anorg. Allg. Chem. 368, 133-143 (1969).

38. Casanova, D. et al. Minimal Distortion Pathways in Polyhedral Rearrangements. J. Am. Chem. Soc. 126, 1755-1763 (2004).

39. Riesen, H. \& Krausz, E. Persistent spectral hole-burning, luminescence line narrowing and selective excitation spectroscopy of the $R$ lines of $\mathrm{Cr}(\mathrm{III}) \operatorname{tris}\left(2,2^{\prime}\right.$-bipyridine) in amorphous hosts. J. Chem. Phys. 97, 7902-7910 (1992).

40. Macfarlane, R. M. High-resolution laser spectroscopy of rare-earth doped insulators: a personal perspective. J. Lumin. 100, 1-20 (2002).

41. Naumov, A. V., Gorshelev, A. A., Vainer, Y. G., Kador, L. \& Köhler, J. Impurity spectroscopy at its 
ultimate limit: relation between bulk spectrum, inhomogeneous broadening, and local disorder by spectroscopy of (nearly) all individual dopant molecules in solids. Phys Chem Chem Phys 13, 1734-1742 (2011).

42. Sigl, A., Orrit, M., Reinot, T., Jankowiak, R. \& Friedrich, J. Terrylene in hexadecane revisited: A hole burning study. J. Chem. Phys. 127, 084510 (2007).

43. Riesen, H. Hole-burning spectroscopy of coordination compounds. Coord. Chem. Rev. 250, 17371754 (2006).

44. de Riedmatten, H., Afzelius, M., Staudt, M. U., Simon, C. \& Gisin, N. A solid-state light-matter interface at the single-photon level. Nature 456, 773-777 (2008).

45. Riesen, H. Progress in Hole-Burning Spectroscopy of Coordination Compounds. in Optical Spectra and Chemical Bonding in Transition Metal Complexes (ed. Schönherr, T.) vol. 107 179-205 (Springer Berlin Heidelberg, 2004).

46. Harley, R. T., Henderson, M. J. \& Macfarlane, R. M. Persistent spectral hole burning of colour centres in diamond. J. Phys. C Solid State Phys. 17, L233-L236 (1984).

47. Naumov, A. V. et al. Micro-Refractometry and Local-Field Mapping with Single Molecules. Nano Lett. 18, 6129-6134 (2018).

48. Casabone, B. et al. Cavity-enhanced spectroscopy of a few-ion ensemble in $\mathrm{Eu}^{3+}: \mathrm{Y}_{2} \mathrm{O}_{3}$. New J. Phys. 20, 095006 (2018).

49. Ahlefeldt, R. L., Manson, N. B. \& Sellars, M. J. Optical lifetime and linewidth studies of the transition in : A potential material for quantum memory applications. J. Lumin. 133, 152-156 (2013).

50. Thiel, C. W., Böttger, T. \& Cone, R. L. Rare-earth-doped materials for applications in quantum information storage and signal processing. J. Lumin. 131, 353-361 (2011).

51. Zhong, T. \& Goldner, P. Emerging rare-earth doped material platforms for quantum nanophotonics. Nanophotonics 8, 2003-2015 (2019).

52. Eremchev, I. Yu., Vainer, Y. G., Naumov, A. V. \& Kador, L. Low-temperature dynamics in 
amorphous polymers and low-molecular-weight glasses-what is the difference? Phys Chem Chem Phys 13, 1843-1848 (2011).

53. Karlsson, J., Kunkel, N., Ikesue, A., Ferrier, A. \& Goldner, P. Nuclear spin coherence properties of ${ }^{151} \mathrm{Eu}^{3+}$ and ${ }^{153} \mathrm{Eu}^{3+}$ in $\mathrm{a}_{2} \mathrm{O}_{3}$ transparent ceramic. J. Phys. Condens. Matter 29, 125501 (2017).

54. Macfarlane, R. M., Genack, A. Z., Weitz, D. A. \& Shelby, R. M. Nuclear-quadrupole optical hole burning in the stoichiometric material EuP_50_14. Opt. Lett. 5, 462 (1980).

55. Nandy, M. et al. Syntheses, structural diversity and photo-physical properties of copper(I) and silver(I) coordination polymers based on the pyridine-4-amidoxime ligand. Polyhedron $65,252-$ 261 (2013).

\section{Data availability}

X-ray crystallographic data of the $\left[\mathbf{E u}_{2}\right]$ and $\left[\mathbf{G d}_{2}\right]$ complexes, in the form of cif file, discussed in this article can be obtained from the Cambridge Crystallographic Data Centre-CCDC $1863020\left[\mathrm{Eu}_{2}\right]$ and $1961893\left[\mathbf{G d}_{2}\right]$. The original data sets corresponding to the experiments can be obtained from the authors upon a reasonable request (contact: mario.ruben@kit.edu).

\section{Acknowledgements}

M.R. thanks the grant agencies innovation FRC, for the financial support for the project Molecular Qudits: Isotopologoues Coordination Chemistry and the DFG priority program 1928 "COORNETS" for generous support. D. S. and P. G. have received funding from the European Union's Horizon 2020 research and innovation programme under grant agreement No 820391 (Square). A.M.N. and L.J.C. thank the French Agence National de la Recherche for financial support (Neutrino project $n^{\circ}$ ANR-16-CE09_0015-02). All the authors thank Dr. Asato Mizuno for the structural refinement of $\left[\mathbf{G d}_{2}\right]$ complex. 


\section{Author contributions}

M.R. and P.G. conceived and supervised the project. K.S. synthesized and characterized the complex and involved in the conceptual development of the project. L.K. determined the Xray structure of the Eu(III) complex. B.H. performed powder X-ray diffraction studies and indexed the patterns. A.M.N. and L.J.C. carried out the steady-state photoluminescence studies. D.S. and P.G. performed PLE and SHB measurements. K.S. D.S. P.G and M.R. wrote the manuscript. All the authors have read and commented on the manuscript.

\section{Additional information}

Supplementary information and chemical compound information are available in the online version of the paper. Reprints and permissions information is available online at www.nature.com/reprints. Correspondence and requests for materials should be addressed to M.R.

\section{Competing financial interests}

The authors declare no competing interests.

\section{Graphical abstract}

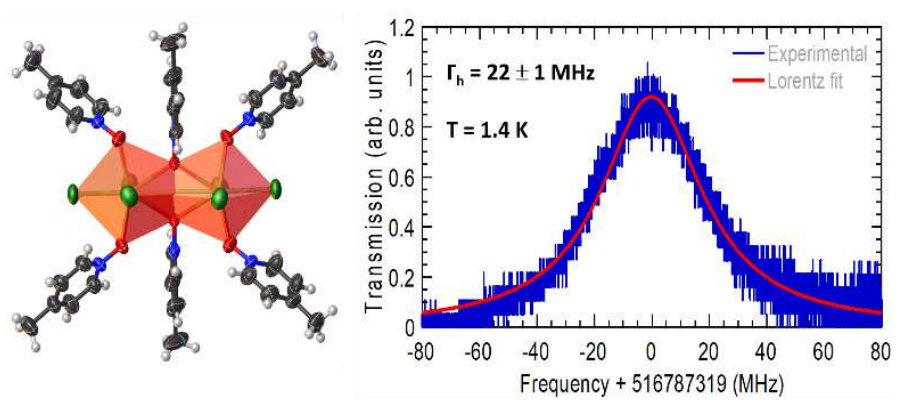

Spectral hole burning (SHB) in the inhomogeneously broadened ${ }^{5} \mathrm{D}_{0} \rightarrow^{7} \mathrm{~F}_{0}$ transition of a binuclear $\mathrm{Eu}(\mathrm{III})$ complex-[ $\left[\mathrm{Eu}_{2}\right]$ - is demonstrated for the first time, paving the way for the 
realization of quantum information processing (QIP) applications based on molecular materials. 


\section{Supporting Information}

\section{Molecule-based coherent light-spin interfaces for quantum information processing - optical spin-state polarization in a binuclear europium complex}

Kuppusamy Senthil Kumar, ${ }^{1,2 *+}$ Diana Serrano, ${ }^{3 *+}$ Aline M. Nonat, ${ }^{4}$ Benoît Heinrich, ${ }^{1}$ Lydia Karmazin, ${ }^{5}$ Loïc J. Charbonnière, ${ }^{4}$ Philippe Goldner, ${ }^{3 *}$ and Mario Ruben ${ }^{2,6,7 *}$

${ }^{1}$ Institut de Physique et Chimie des Matériaux de Strasbourg (IPCMS), CNRS-Université de Strasbourg, 23, rue du Loess, BP 43, 67034 Strasbourg cedex 2, France.

${ }^{2}$ Institute of Nanotechnology, Karlsruhe Institute of Technology (KIT), Hermann-von-Helmholtz-Platz 1, 76344, EggensteinLeopoldshafen, Germany.

${ }^{3}$ Institut de Recherche de Chimie Paris (IRCP), Université PSL, Chimie ParisTech, CNRS, 75005 Paris,

France.

${ }^{4}$ Equipe de Synthèse pour I'Analyse, IPHC, UMR 7178, CNRS-Université de Strasbourg, ECPM, 25 rue Becquerel, 67087 Strasbourg Cedex, France.

${ }^{5}$ Service de Radiocristallographie, Fédération de Chimie Le Bel FR2010 CNRS-Université de Strasbourg, 1 rue Blaise Pascal, BP 296/R8, 67008 Strasbourg cedex, France.

${ }^{6}$ Institute for Quantum Materials and Technologies (IQMT), Karlsruhe Institute of Technology (KIT), Hermann-von-HelmholtzPlatz 1, 76344, Eggenstein-Leopoldshafen, Germany.

${ }^{7}$ Université de Strasbourg (Unistra), Institute de Science et d'Ingénierie Supramoléculaire (ISIS), 8, Allée Gaspard Monge, F67000 Strasbourg, France.

tThese two authors have equally contributed.

*e-mail: senthil.kuppusamy2@kit.edu; diana.serrano@chimieparistech.psl.eu; philippe.goldner@chimieparistech.psl.eu; mario.ruben@kit.edu

\section{Contents}

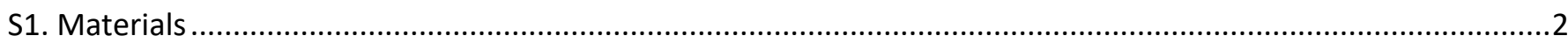

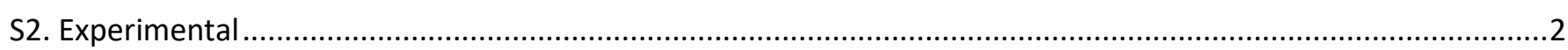

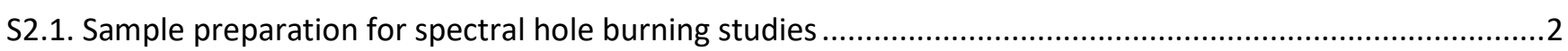

S2.2. Schematic of experimental setup used for spectral hole burning studies ..............................................

S2.3. Preparation of the $\left[\operatorname{Ln}_{2} \mathrm{Cl}_{6}(\operatorname{PicNO})_{4}\left(\boldsymbol{\mu}_{2}-\mathrm{PicNO}\right)_{2}\right] \cdot \mathbf{2} \mathrm{H}_{2} \mathrm{O}\left(\mathrm{Ln}=\mathrm{Eu}\left(\left[\mathrm{Eu}_{2}\right]\right)\right.$ and $\left.\mathrm{Gd}\left(\left[\mathrm{Gd}_{2}\right]\right)\right)$ complexes..............5

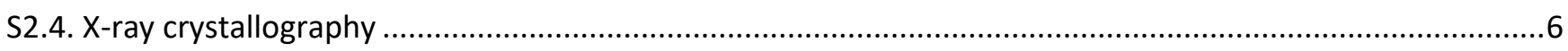

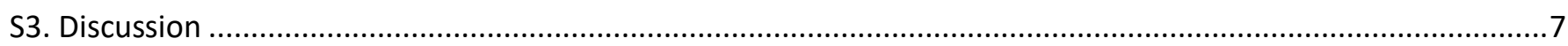

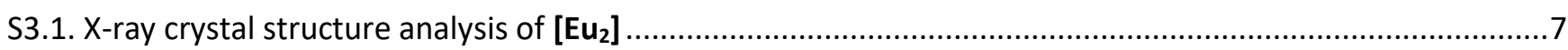


S3.1.1. ORTEP representation and Packing of $\left[\mathrm{Eu}_{2}\right]$ in crystalline state..... 7

S3.1.2 Comparison between the calculated single crystal and powder X-ray diffraction patterns of [Eu $\left.\mathrm{Eu}_{2}\right] . . . . . .8$

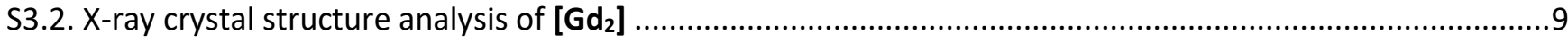

S3.2.1. ORTEP representation and Packing of $\left[\mathbf{G d}_{\mathbf{2}}\right]$ in crystalline state ..................................................10

S3.2.2 Comparison between the calculated single crystal and powder X-ray diffraction patterns of $\left[\mathbf{G d}_{2}\right]$.....11

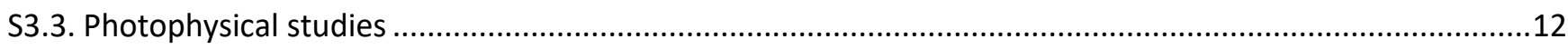

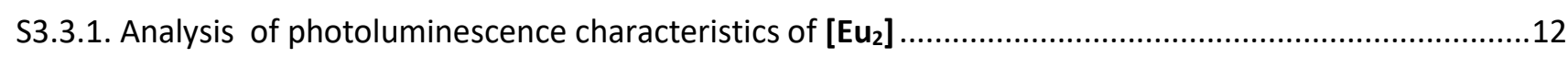

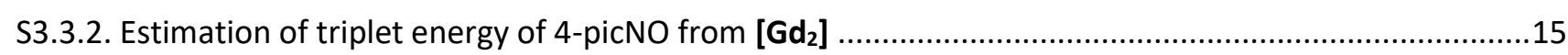

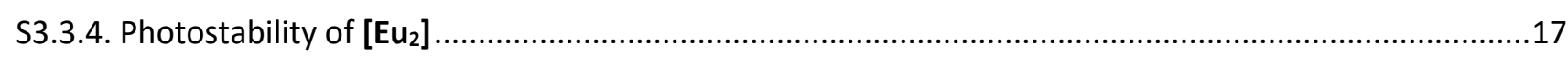

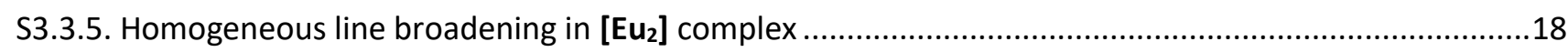

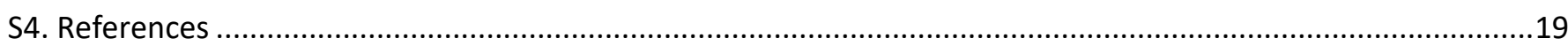

\section{S1. Materials}

The solvents-ethanol (EtOH; absolute) and ethyl acetate (EtOAc; 99.5\%)-used to prepare $\left[\mathrm{Ln}_{2} \mathrm{Cl}_{6}(\mathrm{PicNO})_{4}\left(\boldsymbol{\mu}_{2}-\mathrm{PicNO}\right)_{2}\right] \cdot \mathbf{2} \mathrm{H}_{2} \mathrm{O}(\mathrm{Ln}=\mathrm{Eu}$ and $\mathrm{Gd})$ were purchased from VWR chemicals and used as received. Water was distilled from a double distillation setup. $\mathrm{EuCl}_{3} \cdot 6 \mathrm{H}_{2} \mathrm{O}$ (99.9\% trace metals basis), $\mathrm{GdCl}_{3} \cdot 6 \mathrm{H}_{2} \mathrm{O}$ (99\%; titration), and 4-picoline N-Oxide (98\%) were purchased from Sigma-Aldrich and used as received.

\section{S2. Experimental}

\section{S2.1. Sample preparation for spectral hole burning studies}

For LT spectral hole burning measurements, $5 \mathrm{mg}$ of microcrystalline powder sample (Fig. S1) of $\left[\mathrm{Eu}_{2} \mathrm{Cl}_{6}(\mathrm{PicNO})_{4}\left(\mu_{2}-\mathrm{PicNO}\right)_{2}\right] \cdot 2 \mathrm{H}_{2} \mathrm{O}\left(\left[\mathrm{Eu}_{2}\right]\right)$ was filled into a custom-made sample container $(4 \mathrm{~mm}$ long $\times 2$ $\mathrm{mm}$ wide $\times 0.5 \mathrm{~mm}$ thick) with clear optical openings, as represented in Figure S2 inset. This sample container was then introduced into a He bath cryostat. We note that due to the random orientation of the crystallites in the powder sample, no dependency of the molecular complex spectroscopic features ( $\Gamma_{\text {inh }}$ and or $\Gamma_{h}$ ) is expected with respect to the laser propagation direction nor polarization. 


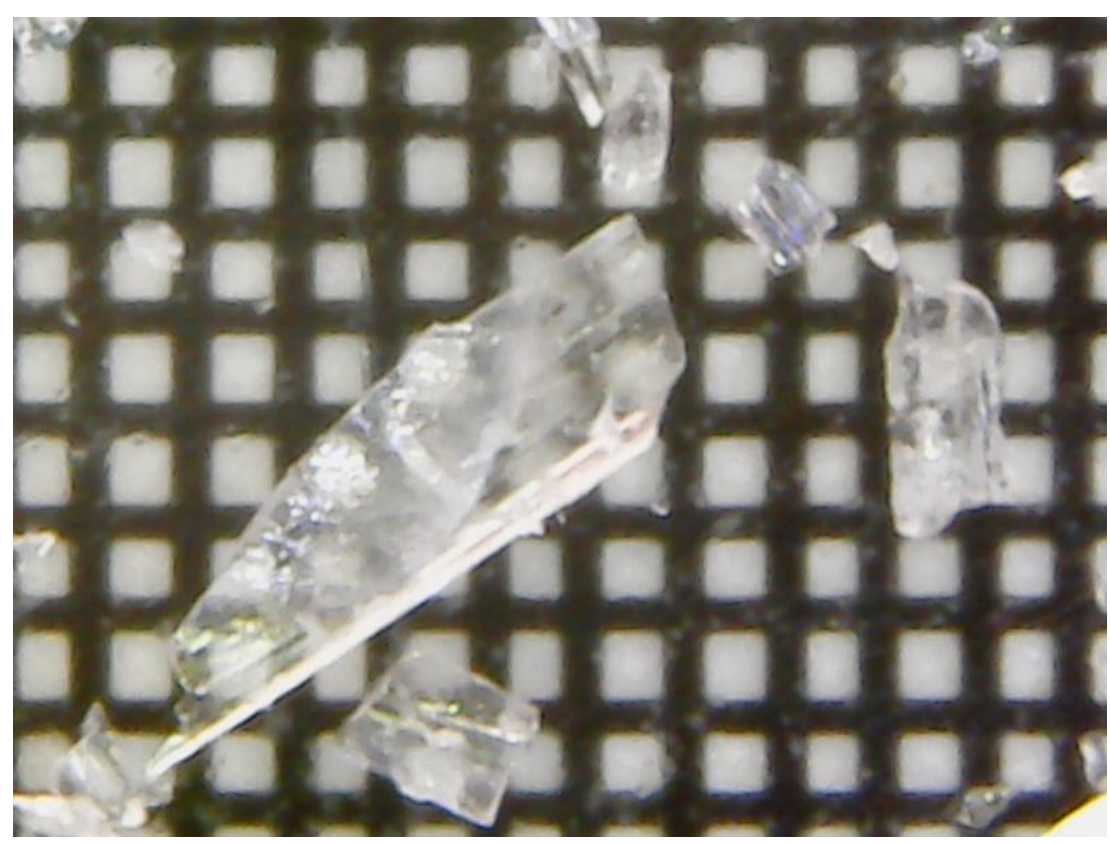

Fig. S1 Optical microscopy image of the $\left[\mathrm{Eu}_{2}\right]$ microcrystalline powder dispersed over a coverslip. Background grid size $=100 \times 100 \mu \mathrm{m}$.

S2.2. Schematic of experimental setup used for spectral hole burning studies

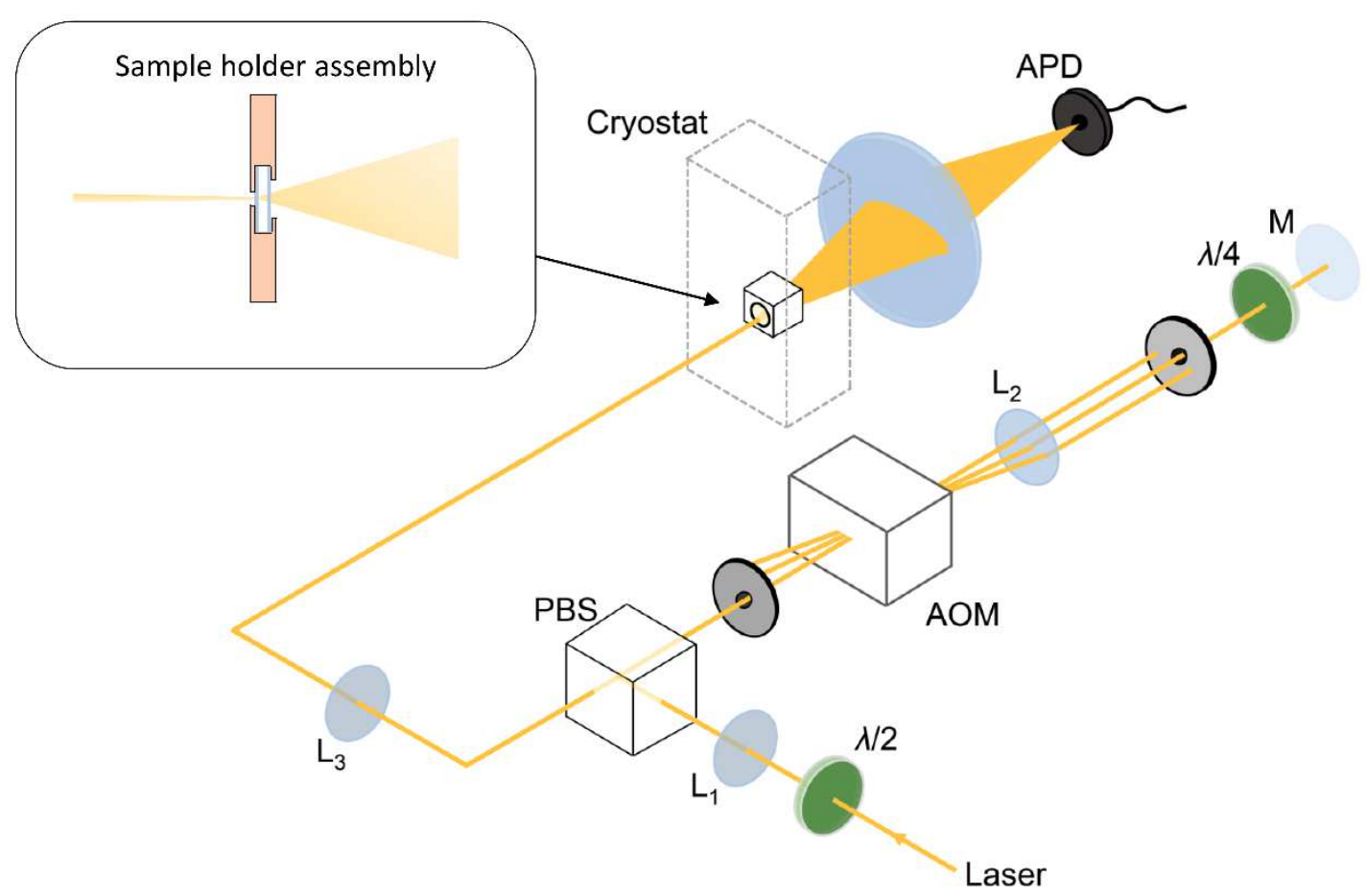

Fig. S2 | Experimental setup. AOM stands for acousto-optic modulator, PBS for polarizing beam splitter and APD for avalanche photo diode, and $M$ for mirror. $L_{n}$ (with $n=1,2,3$ ) are optical lenses. Unlabeled elements correspond to apertures. Inset: Home-built sample holder assembly. 


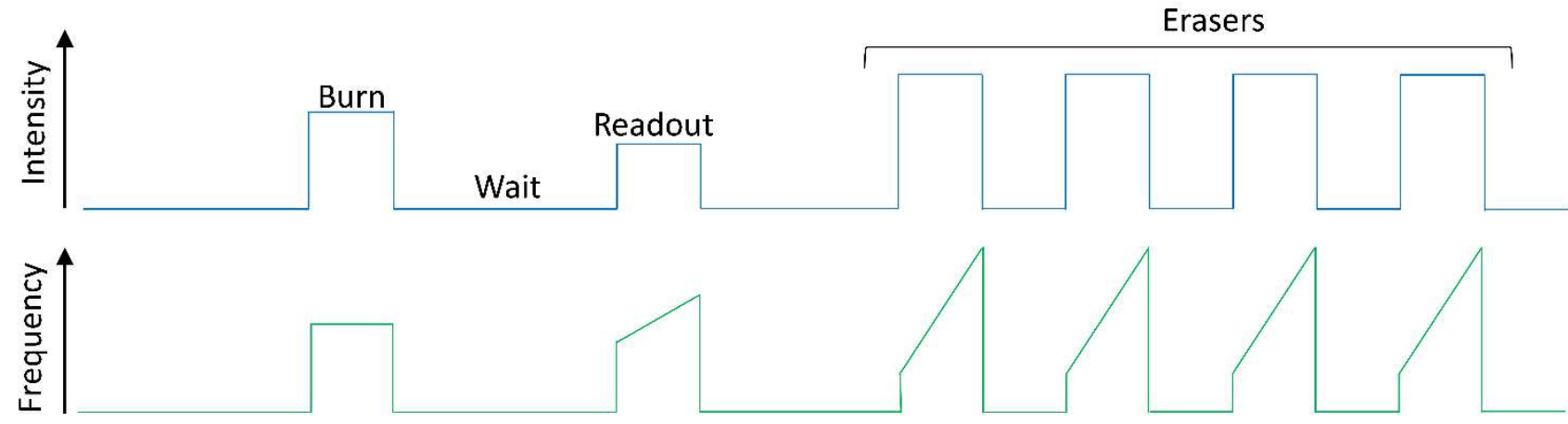

Time

Fig. S3 | Spectral hole burning pulse sequence. Traces show laser intensity and frequency as a function of time. Burning was maintained for $2 \mathrm{~ms}$ and the waiting time before readout was varied from $5 \mathrm{~ms}$ to 10 s. The sequence was ended up by a series of hole erasing pulses. See experimental description for more details.

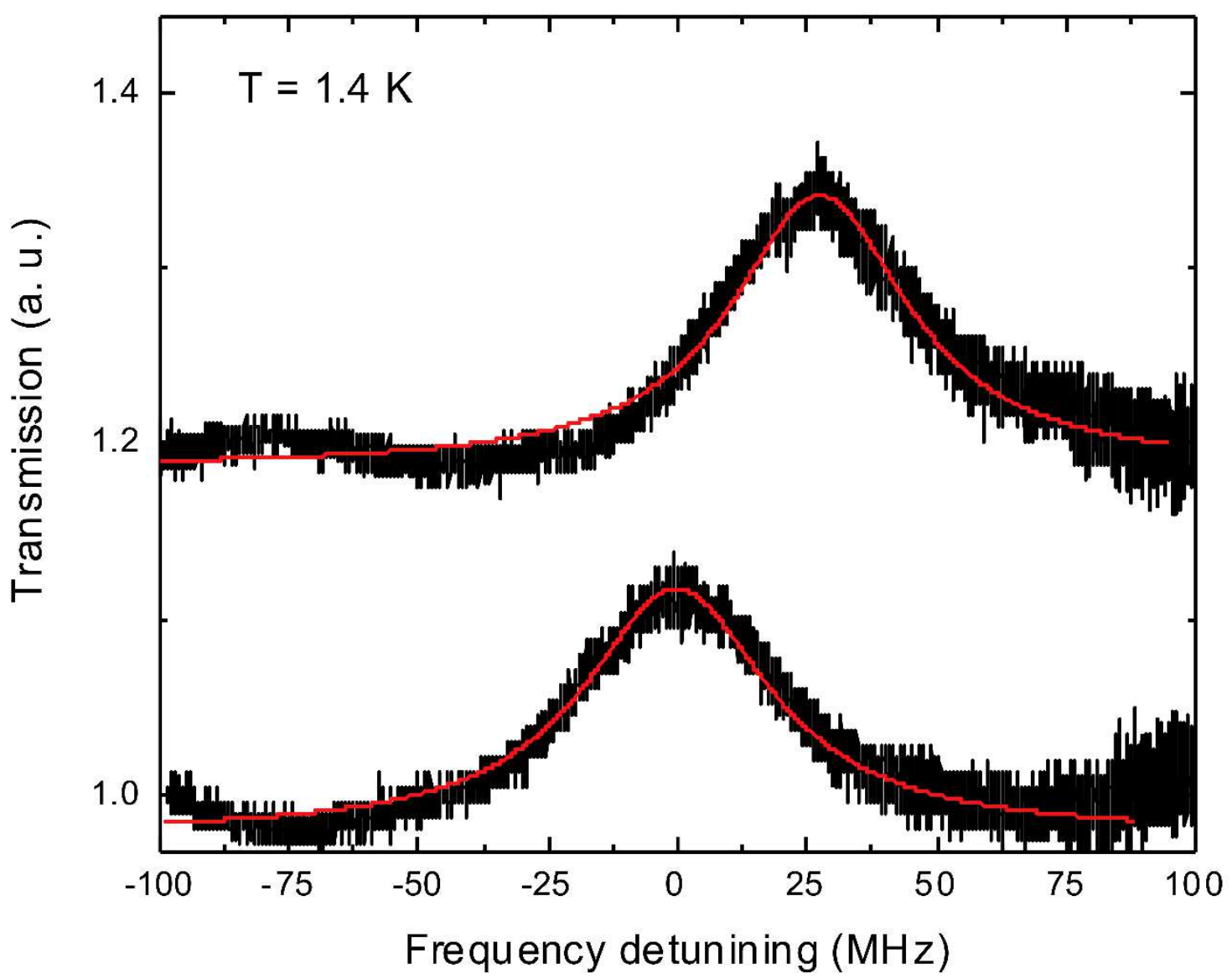

Fig. S4| Spectral holes burned at two frequencies, separated by $25 \mathrm{MHz}$, within the optical inhomogeneous transition. As expected, similar hole width is observed independently of the burning frequency. Data are vertically shifted for clarity. 


\section{S2.3. Preparation of the $\left[\operatorname{Ln}_{2} \mathrm{Cl}_{6}(\mathrm{PicNO})_{4}\left(\mu_{2}-\mathrm{PicNO}\right)_{2}\right] \cdot \mathbf{2} \mathrm{H}_{2} \mathrm{O}\left(\mathrm{Ln}=\mathrm{Eu}\left(\left[\mathrm{Eu}_{2}\right]\right)\right.$ and $\left.\mathrm{Gd}\left(\left[\mathrm{Gd}_{2}\right]\right)\right)$ complexes}

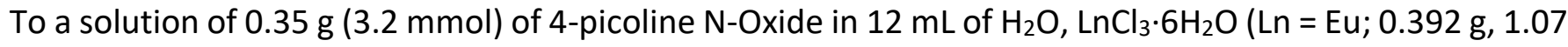
$\mathrm{mmol}$ or $\mathrm{Ln}=\mathrm{Gd} ; 0.397 \mathrm{~g}, 1.07 \mathrm{mmol}$ ) was added as a solid. The reaction mixture was stirred for $10 \mathrm{~min}$ and water was evaporated from the reaction mixture under reduced pressure. The solids were redissolved in $15 \mathrm{~mL}$ of hot EtOH, filtered, and the filtrate was cooled to RT. About $15-20 \mathrm{~mL}$ of EtOAc was carefully added to the filtrate until the formation of a slight precipitate, and the mixture was filtered to obtain a clear solution. The clear solution was left undisturbed for a few days in a closed vial, yielding Xray quality crystals of $\left[\operatorname{Ln}_{2} \mathrm{Cl}_{6}(\text { picNO})_{4}\left(\mu_{2}-\mathrm{picNO}\right)_{2}\right] \cdot \mathbf{2} \mathrm{H}_{2} \mathrm{O}(\mathrm{Ln}=\mathrm{Eu}$ or $\mathrm{Gd})$. The crystalline complexes are stable and can be stored and handled at ambient conditions.
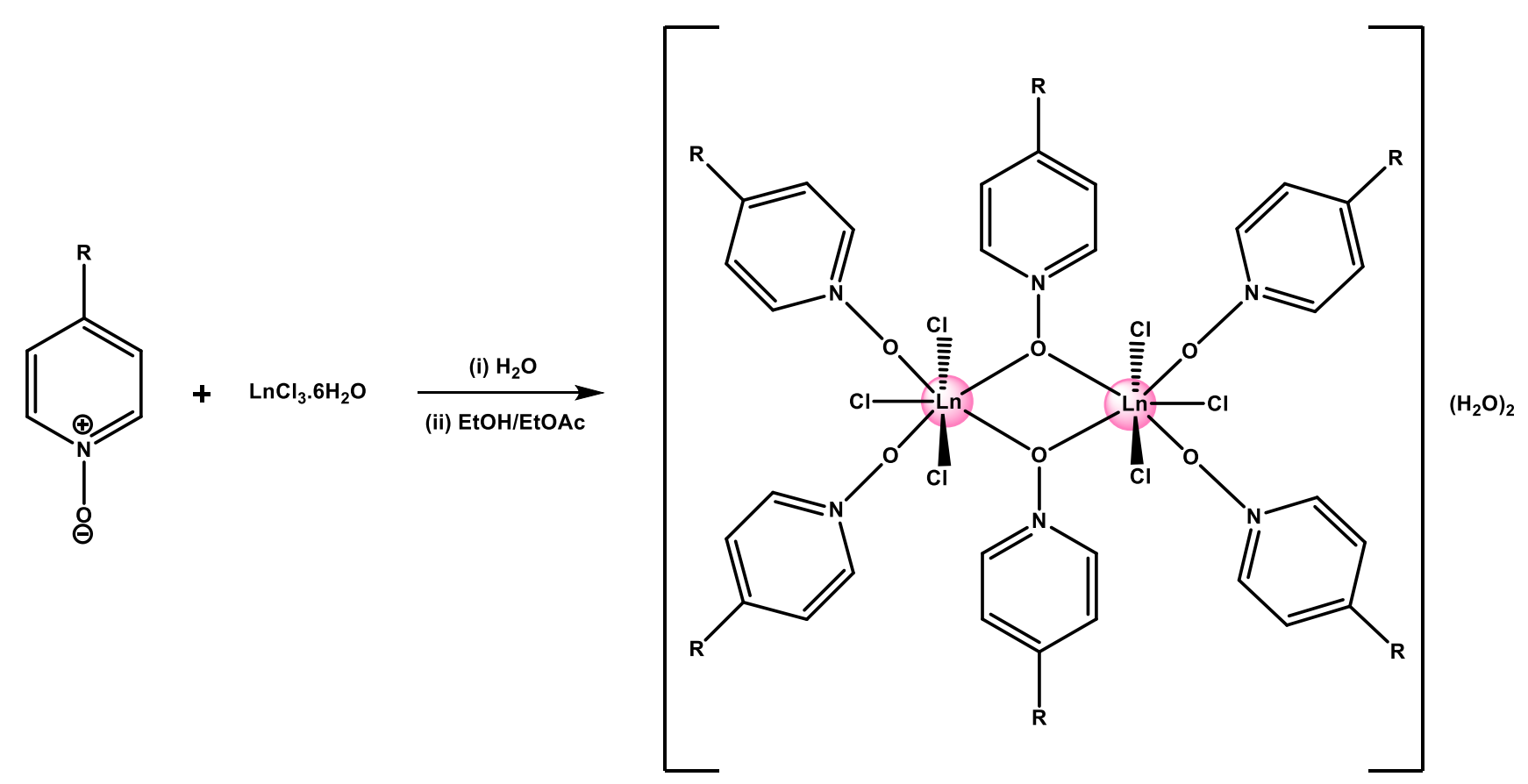

Scheme S1. Preparation of the molecular complexes [Eu $]$ and $\left[\mathrm{Gd}_{2}\right]$. Coordination of three anionic $\mathrm{Cl}^{-}$ ligands with each $\operatorname{Ln}(\mathrm{III})$ center renders the complex charge neutral. The zwitterionic nature of the 4picoline $\mathrm{N}$-Oxide ligand is not shown in the complex structure for clarity. $\mathrm{R}=\mathrm{CH}_{3}$.

[Eu $\mathrm{Cl}_{6}$ (picNO) ${ }_{4}\left(\mu_{2} \text {-picNO) }\right)_{2}$ ] $2 \mathrm{H}_{2} \mathrm{O}$ : Elemental analysis (calcd., found for $\mathrm{C}_{36} \mathrm{H}_{42} \mathrm{Cl}_{6} \mathrm{Eu}_{2} \mathrm{~N}_{6} \mathrm{O}_{6} \cdot 2 \mathrm{H}_{2} \mathrm{O}$ ): C (35.81, 35.74), H (3.84, 3.84), N (6.96; 6.90). Yield: $0.4 \mathrm{~g}(61 \%)$.

$\left[\mathrm{Gd}_{2} \mathrm{Cl}_{6}(\text { picNO})_{4}\left(\boldsymbol{\mu}_{2} \text {-picNO) }\right)_{2}\right] \cdot 2 \mathrm{H}_{2} \mathrm{O}$ : Elemental analysis (calcd., found for $\mathrm{C}_{36} \mathrm{H}_{42} \mathrm{Cl}_{6} \mathrm{Gd}_{2} \mathrm{~N}_{6} \mathrm{O}_{6} \cdot 2 \mathrm{H}_{2} \mathrm{O}$ ): C (35.50, 35.12), H (3.81, 3.79), N (6.90; 6.75). Yield: $0.37 \mathrm{~g}(57 \%)$. 


\section{S2.4. X-ray crystallography}

X-ray diffraction data of [ $\left.\mathrm{Eu}_{2}\right]$ was collected on a Bruker APEX II DUO Kappa-CCD diffractometer equipped with an Oxford Cryosystem liquid $\mathrm{N}_{2}$ device, using Mo-K $\alpha$ radiation $(\lambda=0.71073 \AA$ ). The crystal-detector distance was $38 \mathrm{~mm}$. The cell parameters were determined (APEX3 software; M86-EXX229V1 APEX3 User Manual", Bruker AXS Inc., Madison, USA, 2016) from reflections taken from three sets of 12 frames, each at $10 \mathrm{~s}$ exposure. The structure was solved using the program SHELXT-2014. ${ }^{1}$ The refinement and all further calculations were carried out using SHELXL-2014. ${ }^{2}$ Hydrogen atoms were included in calculated positions and treated as riding atoms using SHELXL default parameters. The non-hydrogen atoms were refined anisotropically, using weighted full-matrix least-squares on $\mathrm{F}^{2}$.

X-ray diffraction data of $\left[\mathbf{G d}_{2}\right]$ complex was collected on a STOE StadiVari 25 diffractometer with a Pilatus300 K detector using GeniX 3D HF micro focus with MoK $\alpha$ radiation $(\lambda=0.71073 \AA)$. The structure was solved using direct methods and was refined by full-matrix least-squares methods on all $F_{2}$ using SHELX-2014 implemented in Olex2. The crystals were mounted on a glass tip using crystallographic oil and placed in a cryostream. Data were collected using $\varphi$ and $\omega$ scans chosen to give a complete asymmetric unit. All non-hydrogen atoms were refined anisotropically. Hydrogen atoms were calculated geometrically riding on their parent atoms. 


\section{S3. Discussion}

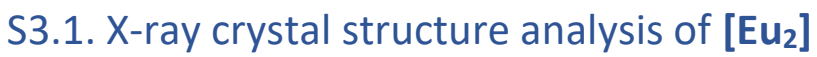

S3.1.1. ORTEP representation and Packing of $\left[\mathrm{Eu}_{2}\right]$ in crystalline state

(a)
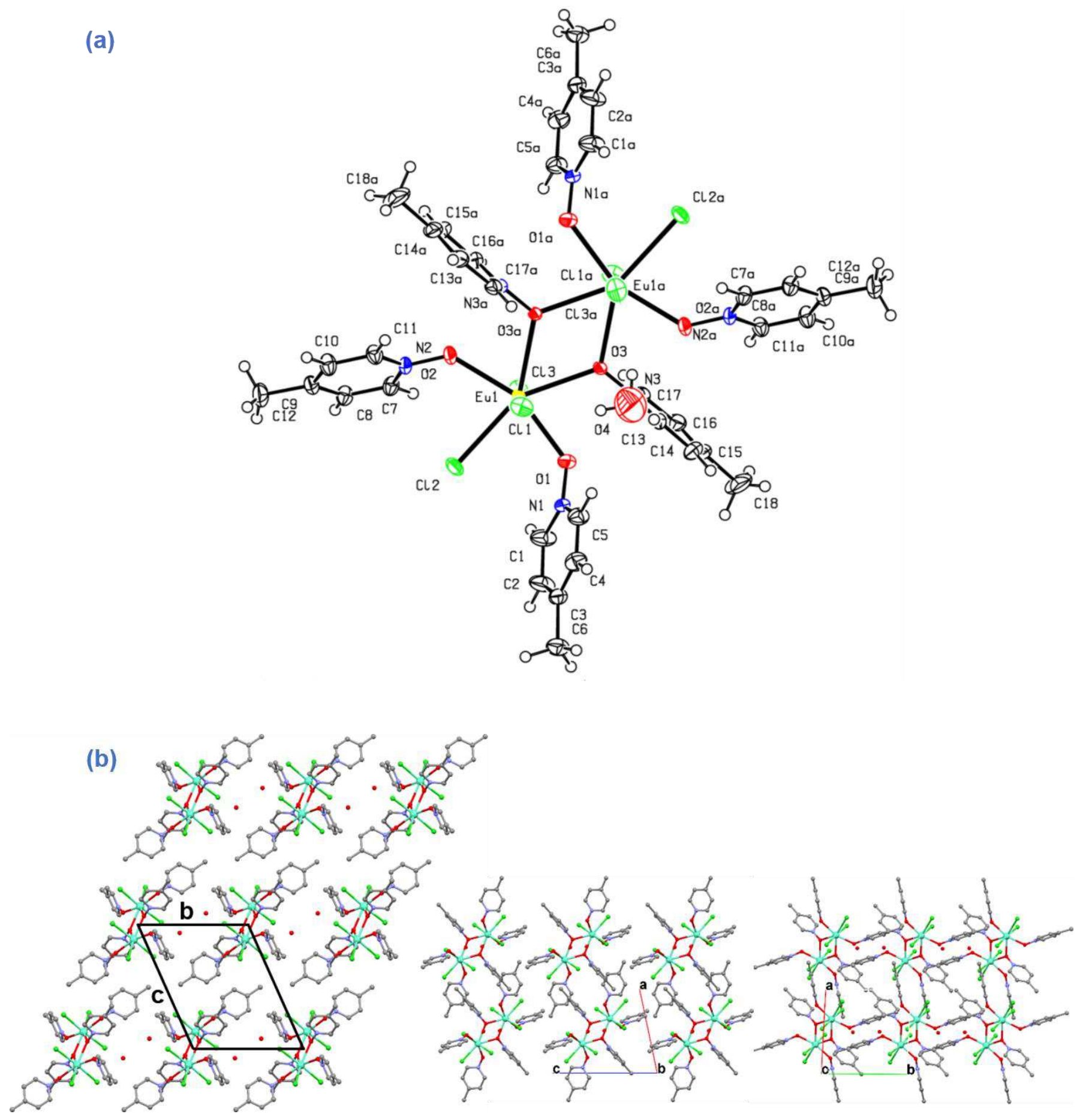

Fig. S5 | (a) ORTEP representation of [ $\left.\mathrm{Eu}_{2}\right]$-The thermal ellipsoids are drawn at a $50 \%$ probability level. (b) Arrangement of [ $\left[\mathrm{Eu}_{2}\right]$ in crystalline state at $173 \mathrm{~K}$, as obtained from single crystal XRD (hydrogen atoms not displayed). Left: view of the bxc plane, in which molecules arrange into layers with two co-crystallized 
water molecules, according to oblique $p 1$ lattice (1 molecule per lattice). Successive layers superpose with constant lateral shift leading to single-layer periodicity and triclinic structure with 1 molecule per cell.

Table S1. Crystallographic data of [Eu $]$

\begin{tabular}{|l|l|l|l|}
\hline Formula & $\mathrm{C}_{36} \mathrm{H}_{42} \mathrm{Cl}_{6} \mathrm{Eu}_{2} \mathrm{~N}_{6} \mathrm{O}_{6} \cdot 2 \mathrm{H}_{2} \mathrm{O}$ & $\mathrm{V} / \AA^{3}$ & $1181.0(2)$ \\
\hline $\mathrm{FW} / \mathrm{g} \cdot \mathrm{mol}^{-1}$ & 1207.41 & $\mathrm{Z}$ & 1 \\
\hline $\mathrm{T} / \mathrm{K}$ & $173 \mathrm{~K}$ & $\rho /{\mathrm{mg} \cdot \mathrm{m}^{-3}}^{-1}$ & 1.698 \\
\hline Crystal System & Triclinic & $\mu / \mathrm{mm}^{-1}$ & 3.022 \\
\hline Space group & $\mathrm{P}-1$ & $\theta$ min-max/ & 1.760 to 30.235 \\
\hline$a / \AA ̊$ & $9.7938(11)$ & Reflns collected & 54056 \\
\hline$b / \AA$ & $10.5255(12)$ & Indep Reflns & 6974 \\
\hline$c / \AA ̊$ & $12.9179(15)$ & Parameters & 265 \\
\hline$\alpha /^{\circ}$ & $66.258(3)$ & GOF on $\mathrm{F}^{2}$ & 1.101 \\
\hline$\beta /^{\circ}$ & $75.915(4)$ & R1 & 0.0436 \\
\hline $\mathrm{V} /{ }^{\circ}$ & $82.000(4)$ & wR2 & 0.1292 \\
\hline
\end{tabular}

S3.1.2 Comparison between the calculated single crystal and powder X-ray diffraction patterns of [Eu 2$]$

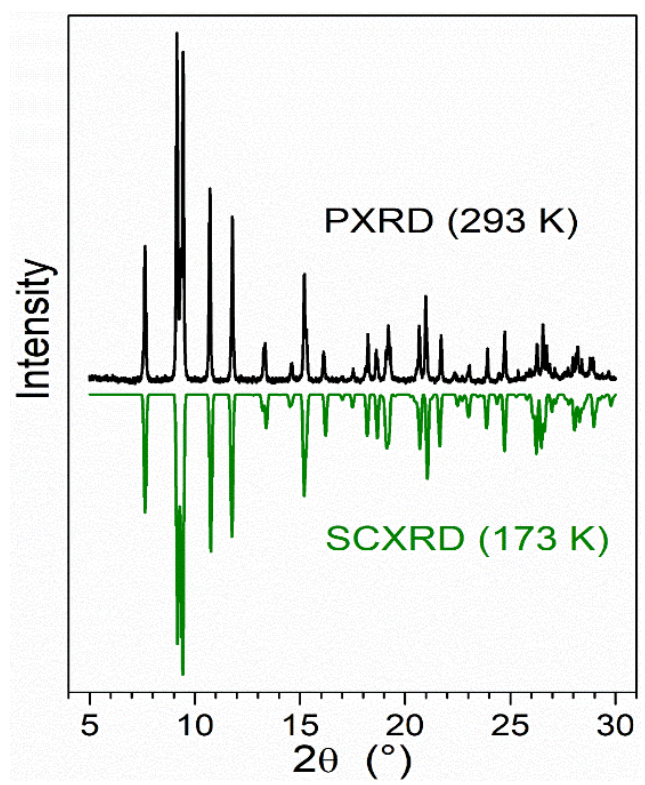

Fig. S6 | Comparison between the powder X-ray diffraction (PXRD) and the calculated single crystal X-ray

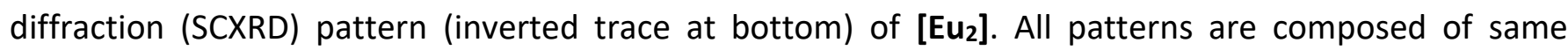
reflections with only tiny changes in peak position and intensity, in relation with tiny variations of lattice parameters and electron densities. 


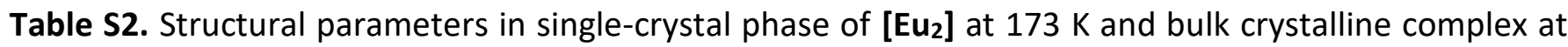
293 K. Parameters obtained from SCXRD and PXRD data are nearly identical at both temperatures within a volume expansion at the limit of significance ( $0.1 \%)$ and an unchanged lateral shift between successive layers ( 3.19 $\AA$ ); the tiny $\sqrt{ } A / d$ ratio increase $(\sim 0.2 \%)$ reveals a very slight lateral expansion of layers.

\begin{tabular}{|c|c|c|}
\hline$M$ & $\mathrm{~T}(\mathrm{~K})$ & $\begin{array}{l}\left.\text { Lattice parameters }(\AA)^{\circ}\right) \text {, Cell Volume } V\left(\AA^{3}\right) \text { [ } Z=1 \text { molecule par lattice] } \\
\text { bxc sublattice area } A\left(\AA^{2}\right) \text {, layer spacing } d=d_{100}(\AA ̊)\end{array}$ \\
\hline $\begin{array}{l}\text { SC- } \\
\text { XRD }\end{array}$ & 173 & $\begin{array}{l}a=9.7938, b=10.5255, c=12.9179, \alpha=66.258, \beta=75.915, \gamma=82.000 \\
V=1181.02, A=124.46, d=9.4891, \sqrt{ } A / d=1.1757\end{array}$ \\
\hline PXRD & 293 & $\begin{array}{l}a=9.79(32), b=10.52(56), c=12.90(85), \alpha=66.57, \beta=75.65, \gamma=82.29 \\
V=1181.84, A=124.6(7), d=9.48, \sqrt{ } A / d=1.178\end{array}$ \\
\hline
\end{tabular}

\section{S3.2. X-ray crystal structure analysis of $\left[\mathbf{G d}_{2}\right]$}

(a)

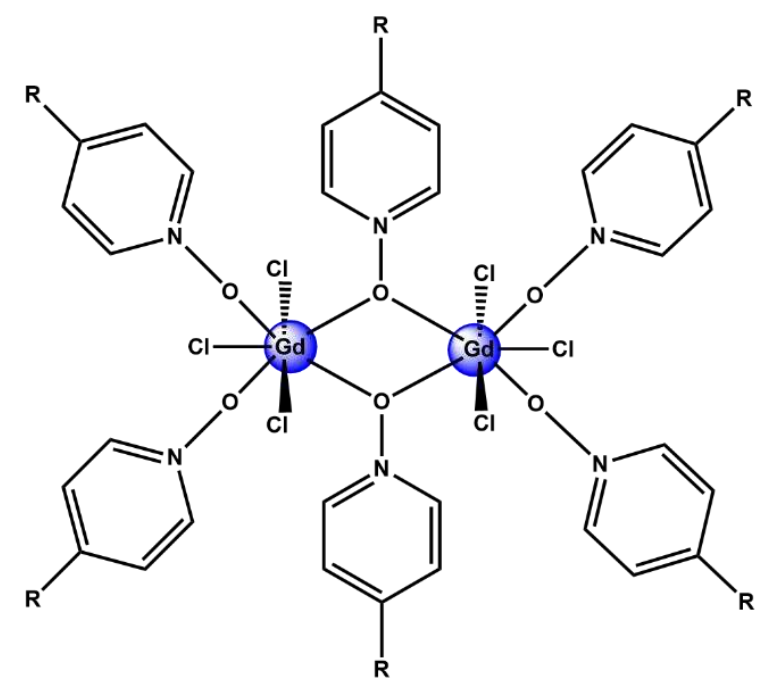

(b)

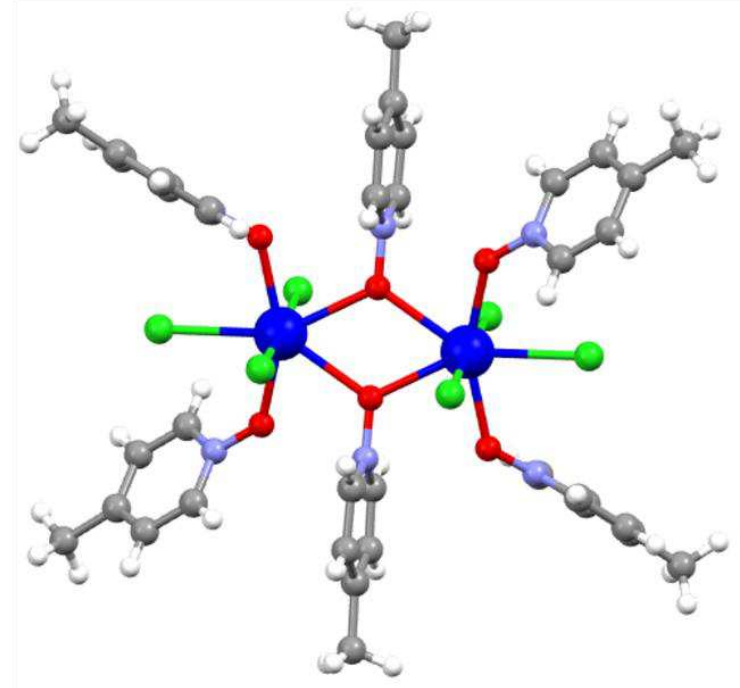

Fig. S7 | Structure of $\left[\mathrm{Gd}_{2}\right]$. (a) Molecular structure of the complex showing the ligands $\left(\mathrm{R}=\mathrm{CH}_{3}\right)$ coordinating with the $\mathrm{Gd}(\mathrm{III})$ centers. (b) X-ray crystal structure of the complex. Coordination geometry around each Eu(III) centre of the complex is best described as pentagonal bipyramidal. The cocrystallized water molecules are omitted for clarity. Colour code: $\mathrm{H}$, white; $\mathrm{C}$, grey; $\mathrm{N}$, blue; $\mathrm{Cl}$, green; Gd, blue. 
(a)

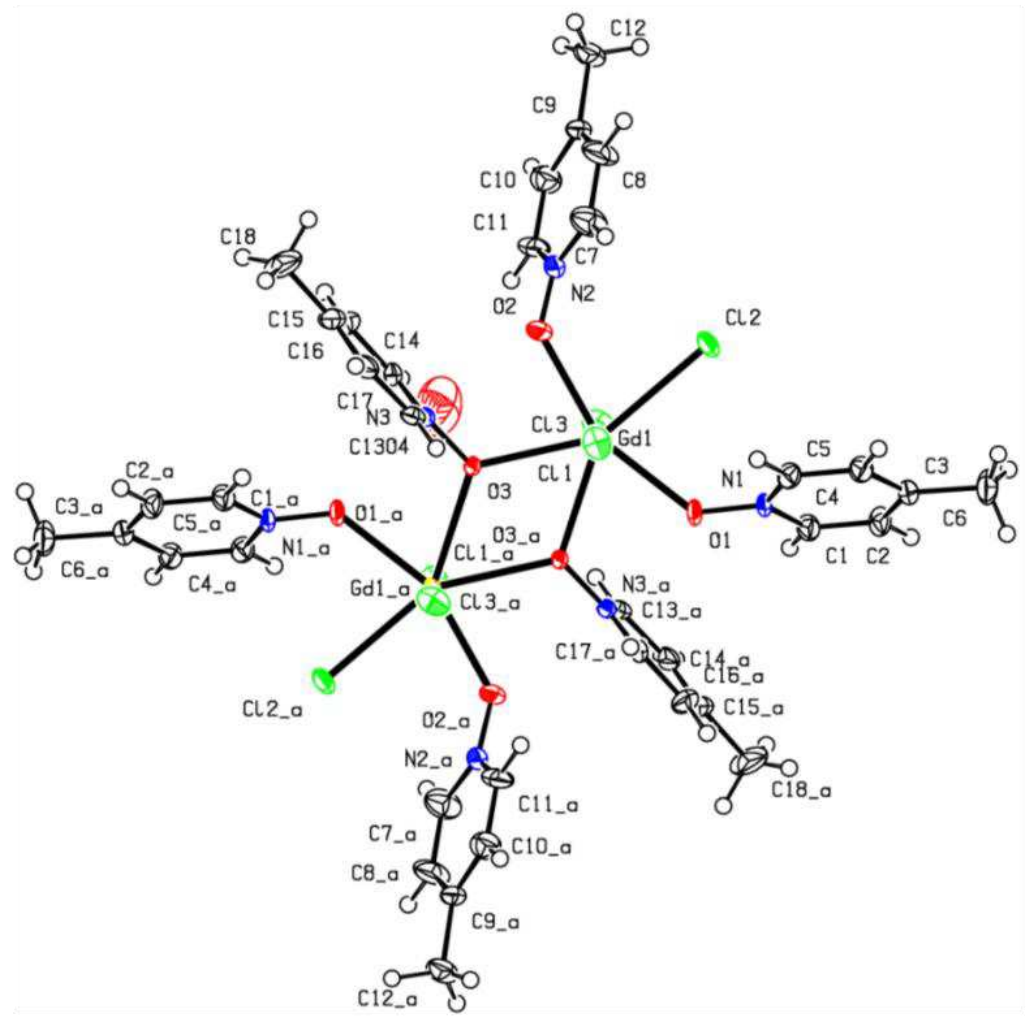

(b)

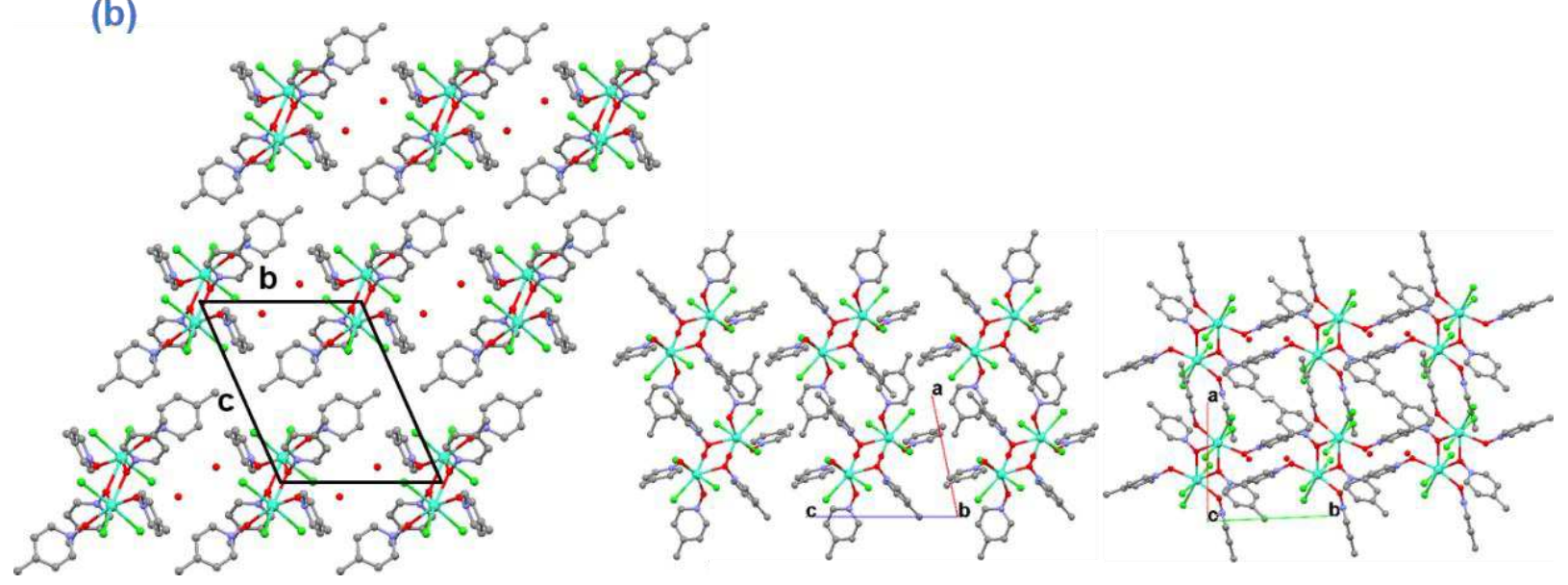

Fig. S8 | (a) ORTEP representation of [ $\left.\mathbf{G d}_{\mathbf{2}}\right]$-The thermal ellipsoids are drawn at a 50\% probability level. (b) Arrangement of $\left[\mathbf{G d}_{\mathbf{2}}\right]$ in crystalline state at $180 \mathrm{~K}$, as obtained from single-crystal XRD (hydrogen atoms not displayed). Left: view of the bxc plane, in which molecules arrange into layers with two co-crystallized water molecules, according to oblique $p 1$ lattice (1 molecule per lattice). Successive layers superpose with constant lateral shift leading to single-layer periodicity and triclinic structure with 1 molecule par cell. 
Table S3. Crystallographic data of $\left[\mathbf{G d}_{2}\right]$

\begin{tabular}{|l|l|l|l|}
\hline Formula & $\mathrm{C}_{36} \mathrm{H}_{42} \mathrm{Cl}_{6} \mathrm{Gd}_{2} \mathrm{~N}_{6} \mathrm{O}_{6} \cdot 2 \mathrm{H}_{2} \mathrm{O}$ & $\mathrm{V} / \AA^{3}$ & 1166.85 \\
\hline $\mathrm{FW} / \mathrm{g} \cdot \mathrm{mol}^{-1}$ & 1213.96 & $\mathrm{Z}$ & 1 \\
\hline $\mathrm{T} / \mathrm{K}$ & $180 \mathrm{~K}$ & $\rho / \mathrm{mg}^{-3}$ & 1.728 \\
\hline Crystal System & Triclinic & $\mu / \mathrm{mm}^{-1}$ & 3.213 \\
\hline Space group & $\mathrm{P}-1$ & $\theta$ min-max/ & $2.9370-27.8190$ \\
\hline$a / \AA$ & 9.7768 & Reflns collected & 13777 \\
\hline$b / \AA$ & 10.4291 & Indep Reflns & 5505 \\
\hline$c / \AA$ & 12.8331 & Parameters & 265 \\
\hline$\alpha /^{\circ}$ & $66.978(5)$ & GOF on $\mathrm{F}^{2}$ & 1.105 \\
\hline$\beta /^{\circ}$ & $75.885(5)$ & R1 & 0.0330 \\
\hline $\mathrm{V} /{ }^{\circ}$ & $82.310(5)$ & wR2 & 0.0880 \\
\hline
\end{tabular}

S3.2.2 Comparison between the calculated single crystal and powder X-ray diffraction patterns of $\left[\mathrm{Gd}_{2}\right]$

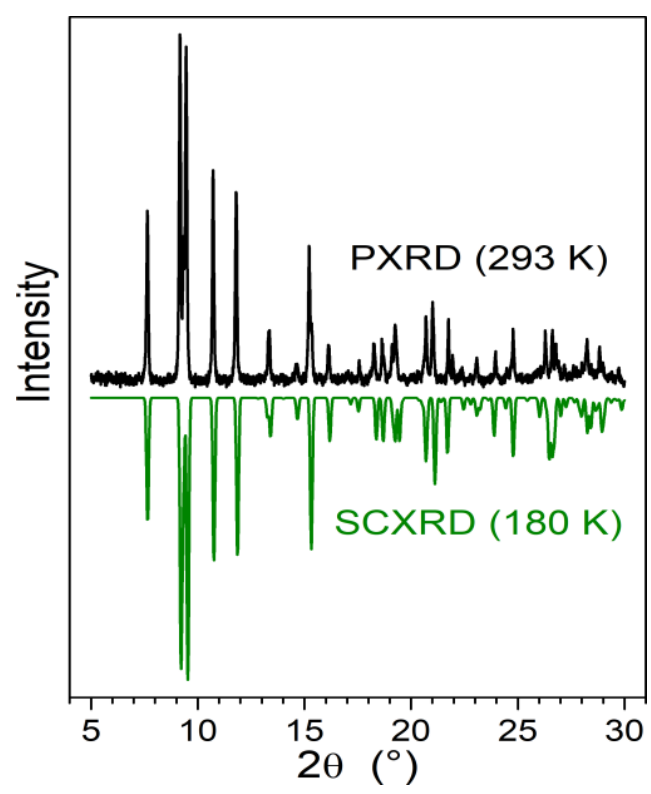

Fig. S9| Comparison between the powder X-ray diffraction (PXRD) and the calculated single crystal X-ray diffraction (SCXRD) pattern (inverted trace at bottom) of $\left[\mathbf{G d}_{2}\right]$. All patterns are composed of same reflections with only tiny changes in peak position and intensity, in relation with tiny variations of lattice parameters and electron densities. 
Table S4. Structural parameters in single-crystal phase of $\left[\mathbf{G d}_{\mathbf{2}}\right]$ at $173 \mathrm{~K}$ and bulk crystalline complex at 293 K. Parameters obtained from SCXRD and PXRD data are nearly identical at both temperatures within a volume expansion at the limit of significance ( $0.1 \%)$ and an unchanged lateral shift between successive layers ( 3.19 $\AA$ ); the tiny $\sqrt{ } A / d$ ratio increase ( $0.2 \%)$ reveals a very slight lateral expansion of layers.

\begin{tabular}{|l|l|l|}
\hline M & T (K) & $\begin{array}{l}\text { Lattice parameters }\left(\AA{ }^{\circ}\right), \text { Cell Volume } V\left(\AA^{3}\right)[Z=1 \text { molecule par lattice }] \\
\text { bxc sublattice area } A\left(\AA^{2}\right) \text {, layer spacing } d=d_{100}(\AA)\end{array}$ \\
\hline SC- & 180 & $a=9.7768, b=10.4291, c=12.8331, \alpha=66.978, \beta=75.885, \gamma=82.310$ \\
\hline XRD & $V=1166.22, A=123.14, d=9.4712, \sqrt{ } A / d=1.1716$ \\
\hline PXRD & 293 & $a=9.78(63), b=10.51(23), c=12.88(72), \alpha=66.60, \beta=75.57, \gamma=82.27$ \\
& $V=1177.40, A=124.3(3), d=9.47, \sqrt{ } A / d=1.177$
\end{tabular}

\section{S3.3. Photophysical studies}

\section{S3.3.1. Analysis of photoluminescence characteristics of $\left[\mathrm{Eu}_{2}\right]$}

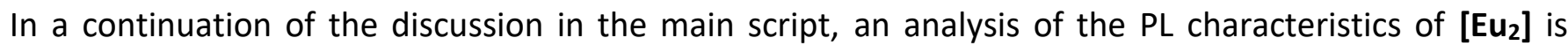
presented in the following sections. The near infra-red (NIR) ${ }^{5} \mathrm{D}_{0} \rightarrow{ }^{7} \mathrm{~F}_{5,6}$ transitions were seen around 745 $\mathrm{nm}\left({ }^{5} \mathrm{D}_{0} \rightarrow{ }^{7} \mathrm{~F}_{5}\right)$ and in the $801 \mathrm{~nm}$ to $836 \mathrm{~nm}\left({ }^{5} \mathrm{D}_{0} \rightarrow{ }^{7} \mathrm{~F}_{6}\right)$ region. The ${ }^{5} \mathrm{D}_{0} \rightarrow{ }^{7} \mathrm{~F}_{5}$ and ${ }^{5} \mathrm{D}_{0} \rightarrow^{7} \mathrm{~F}_{6}$ transitions amount to $3.3 \%$ and $7.5 \%$, respectively, of the total emission intensity. Remarkably, the ${ }^{5} D_{0} \rightarrow{ }^{7} F_{4}$ transition centered around $700 \mathrm{~nm}$ accounts for $26 \%$ of the total emission. Overall, a deep red emission with chromaticity coordinates $(x=0.6615, y=0.3382)$ on the commission internationale de l'éclairage $(\mathrm{CIE})$ color space (Fig. S10a) was observed for the complex. A strictly mono-exponential decay, confirming the presence of a single emitting Eu(III) species, of luminescence was obtained upon monitoring decay at 616 nm (Fig. S10b).

It is well documented that the ${ }^{5} D_{0} \rightarrow{ }^{7} F_{2,4,6}$ transitions are allowed electric-dipole transitions, which are strongly influenced by the crystal field.

The emission spectrum of the $\left[\mathrm{Eu}_{2}\right]$ is in agreement with the low coordination symmetry of the complex, as determined from the X-ray crystallography study. First of all, the ${ }^{5} D_{0} \rightarrow^{7} F_{0}$ transition, which is essential for SHB in the present studies, is unambiguously seen at $580 \mathrm{~nm}$. In addition, the degree of distortion 
around $\mathrm{Eu}(\mathrm{III})$ - as evaluated from the measurement of the intensity ratio $\mathrm{I}\left({ }^{5} \mathrm{D}_{0} \rightarrow{ }^{7} \mathrm{~F}_{2}\right) / 1\left({ }^{5} \mathrm{D}_{0} \rightarrow{ }^{7} \mathrm{~F}_{1}\right)$ was found to be $\mathrm{R}_{21}=7.6$ and reveals a distorted coordination environment or low-site symmetry around the $\mathrm{Eu}(\mathrm{III})$ centers in the complex. The rationale behind this approach is that the magnetic dipolar ${ }^{5} D_{0} \rightarrow^{7} F_{1}$ transition is not influenced by the crystal field, whereas the electric dipolar ${ }^{5} D_{0} \rightarrow^{7} F_{2}$ transition is strongly influenced (hypersensitive) by the local symmetry of the electric/crystal field around a Eu(III) center. Finally, the intensity ratio $I\left({ }^{5} D_{0} \rightarrow{ }^{7} F_{4}\right) / I\left({ }^{5} D_{0} \rightarrow{ }^{7} F_{2}\right)$ is smaller than one, as expected in a low symmetry environment. $A$

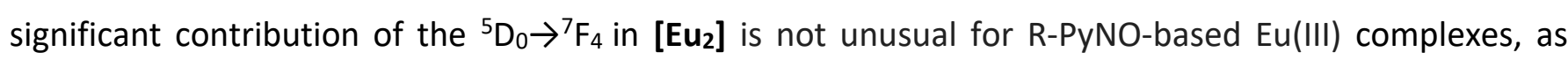
reported in the literature before. ${ }^{3}$ This is due to the high polarizability of R-PyNO ligands, bringing an unusually high contribution of the ligand polarizability-dependent dynamic coupling mechanism (versus electric dipole mechanism) according to Judd-Ofelt theory.
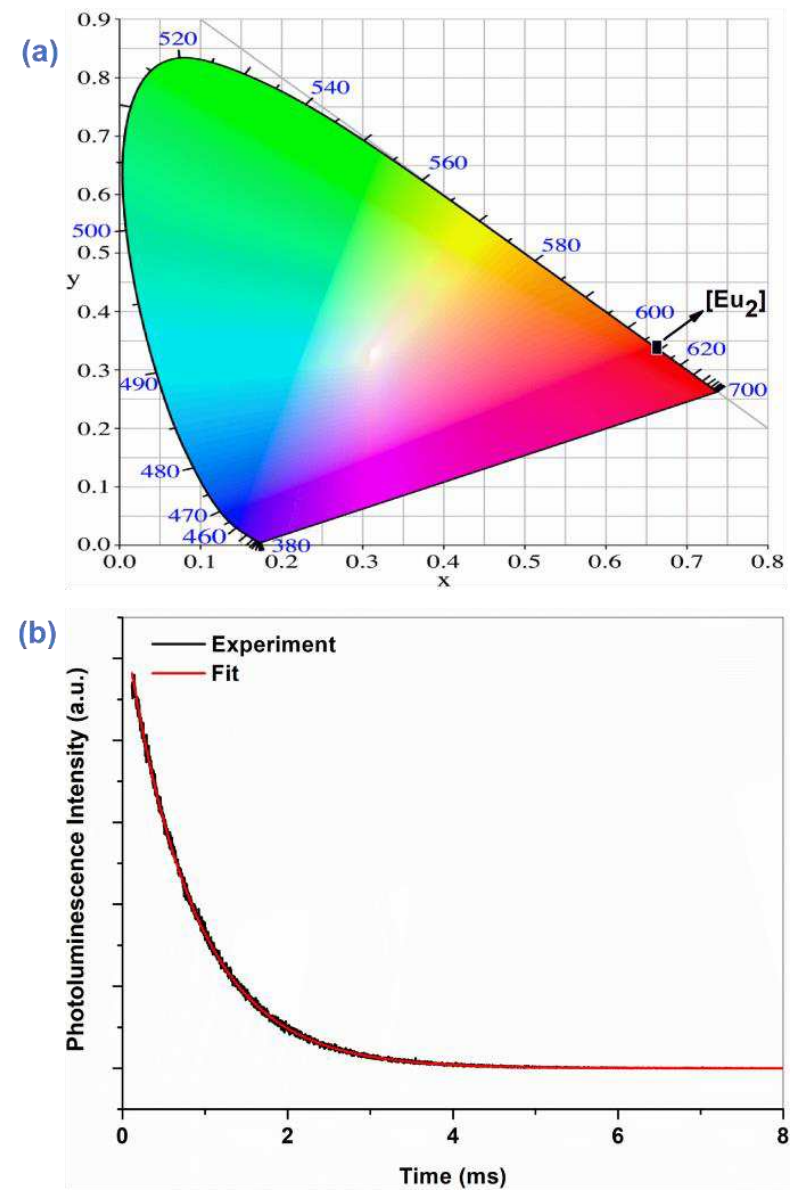

Fig. S10 | (a) The CIE chromaticity diagram of [Eu $]$ in the solid state: $x=0.6615, y=0.3382$, and (b) Luminescence decay of the ${ }^{5} \mathrm{D}_{0}$ excited state of the Eu(III) complex measured at $R T$, the fluorescence decay is well fitted by a single exponential function, suggesting a single emitting Eu(III) centre. 
A PL quantum yield of $38 \pm 6 \%$ was experimentally determined for the complex. The total PL quantum yield, $Q_{\text {tot, }}$ can be represented as the product of the sensitization efficiency $\left(\eta_{\text {sens }}\right)$ of the ligand and the intrinsic quantum yield $\left(Q_{E U}\right)$ of the europium emission from the ${ }^{5} D_{0}$ level:

$Q_{\text {tot }}=\eta_{\text {sens }} \times Q_{E u}$
$Q_{E u}=\tau_{\mathrm{obs}} / \tau_{\mathrm{rad}}$

Where $\tau_{\text {rad }}$ is the radiative lifetime of the ${ }^{5} D_{0}$ level. The radiative lifetime is expressed as

$\tau_{\text {rad }}=\left[\mathrm{A}_{\mathrm{MD}} \times(\mathrm{n})^{3} \times \mathrm{I}_{\mathrm{tot}} / \mathrm{I}_{\mathrm{MD}}\right]^{-1}$

Where $A_{M D}$ is the spontaneous emission probability for the ${ }^{5} D_{0} \rightarrow{ }^{7} F_{1}$ transition in vacuum (taken here as $14.65 \mathrm{~s}^{-1}$ ), $\mathrm{n}$ is the refractive index (taken here as 1.5) of the solid complex, and $\mathrm{I}_{\mathrm{tot}} / \mathrm{I}_{\mathrm{MD}}$ is the ratio of the total integrated emission intensity (for all transitions from the ${ }^{5} \mathrm{D}_{0}$ state to the ${ }^{7} \mathrm{~F}_{\mathrm{J}}$ manifold (Itot)) to that of the magnetic dipole ${ }^{5} D_{0} \rightarrow^{7} F_{1}$ transition $\left(I_{M D}\right) ; I_{\text {tot }} / I_{M D}=14.95$ is obtained from the spectrum depicted in Fig. $2 b$. The values of $\tau_{\text {rad }}=1353 \mu \mathrm{s}$ and $\eta_{\text {sens }}=\sim 62 \%$ were calculated from equations 3 and 1 , respectively. An intrinsic Eu quantum yield $\left(Q_{E u}\right)=\sim 61 \%$ was obtained from equation 2.

The obtained values of $\tau_{\mathrm{rad}}$ and $\tau_{\mathrm{obs}}$ are used to estimate the competition between the radiative and nonradiative processes, which in turn determines $Q_{\mathrm{Eu}}$. While $\tau_{\mathrm{rad}}$ is not influenced by the non-radiative processes, such processes influence $\tau_{\mathrm{obs}}$. Thus, the rate constants for radiative $\left(\mathrm{k}_{\mathrm{r}}\right)$ and non-radiative $\left(\mathrm{k}_{\mathrm{nr}}\right)$ processes can be estimated from equations 4 and 5 .

$\mathrm{k}_{\mathrm{r}}=1 / \tau_{\mathrm{rad}}$

and

$k_{n r}=\left[\left(1 / \tau_{\text {obs }}\right)-\left(k_{r}\right)\right]$

The obtained $\mathrm{k}_{\mathrm{r}}=739 \mathrm{~S}^{-1}$ and $\mathrm{knr}_{\mathrm{n}}=477 \mathrm{~S}^{-1}$ indicate the role of competitive non-radiative relaxation process-most probably, C-H vibrations arising from the 4-picNO ligand skeletons-in reducing $\mathrm{Q}_{\mathrm{Eu}}$.

Apart from $Q_{E u}$, the total sensitization (energy transfer) efficiency $\left(\eta_{\text {sens }}\right)$ of ligand also plays a crucial role in determining the overall luminescence quantum yield $\left(Q_{t o t}\right)$ of $\mathrm{Eu}(I I I)$ luminescence upon excitation of the light absorbing ligand. The sensitization efficiency of a ligand depends on the energy separation $(\Delta E)$ between the triplet state $\left(E_{T}\right)$ of the ligand and $E u(I I I)$ acceptor state $\left({ }^{5} D_{0}\right)$. An energy gap of $2500 \mathrm{~cm}^{-1}<\Delta E\left({ }^{3} \pi \pi-{ }^{5} D_{0}\right)<3500 \mathrm{~cm}^{-1}$ is proposed optimal for the luminescence sensitization of $\mathrm{Eu}(\mathrm{III})$. A non-optimal $\Delta \mathrm{E}=\sim 6579 \mathrm{~cm}^{-1}$ has been estimated for [ $\left[\mathrm{Eu}_{2}\right]$, which justifies the $\eta_{\text {sens }}=\sim 62.54 \%$ calculated for 4-picNO. Note, $E_{T}=23809 \mathrm{~cm}^{-1}$ has been estimated for 4-picNO (see section S3.3.2 and Fig. S11) from the phosphorescence emission of $\left[\mathbf{G d}_{2}\right]$ at $77 \mathrm{~K}$. 
Despite the presence of non-radiative pathways and non-optimal $\Delta E,\left[\mathrm{Eu}_{2}\right]$ complex featured $\sim 38 \pm 6 \%$ total luminescence quantum yield. A sensitization mechanism involving direct funnelling of energy from the triplet level of 4-PicNO to ${ }^{5} D_{J}(J=2,1$, and 0$)$ levels and subsequent radiative relaxation of the ${ }^{5} D_{0}$ level to ground-state J multiplets (Fig. 2a) caused the Eu(III) centred emission in [ $\left.\mathrm{Eu}_{2}\right]$.

The optimal energy gap of $2500 \mathrm{~cm}^{-1}<\Delta E\left({ }^{3} \pi \pi-{ }^{5} D_{0}\right)<3500 \mathrm{~cm}^{-1}$ required for the efficient sensitization of Eu(III) luminescence could be achieved by reducing ${ }^{3} \pi \pi$ energy of the 4-picNO ligand by replacing the methyl group with an electron withdrawing group-such as $\mathrm{CCl}_{3}$.

\section{S3.3.2. Estimation of triplet energy of 4-picNO from $\left[\mathrm{Gd}_{2}\right]$}

To assess the triplet state energy of the 4-picNO ligand involved in the Eu(III) luminescence sensitization process, PL spectra of $\left[\mathbf{G d}_{2}\right]$ was measured upon excitation $\left(\lambda_{\text {ex }}\right)$ of 4-picNO at $291 \mathrm{~nm}$. A broad and structured emission band with $\lambda_{\max }=492 \mathrm{~nm}$ was observed (Fig. S11). By considering the lowest energy fine structure located at $\sim 20 \mathrm{~nm}$, a triplet energy $\left(E_{T}\right)=23809 \mathrm{~cm}^{-1}$ is obtained for 4-picNO, which is in agreement with the 0 -phonon component determined from the intersection of the $x$ axis and the tangent line at $I_{\max / 2}$. The ${ }^{3} \pi \pi^{*}$ excited state decayed mono-exponentially with a lifetime of $1.08 \mathrm{~ms}$.

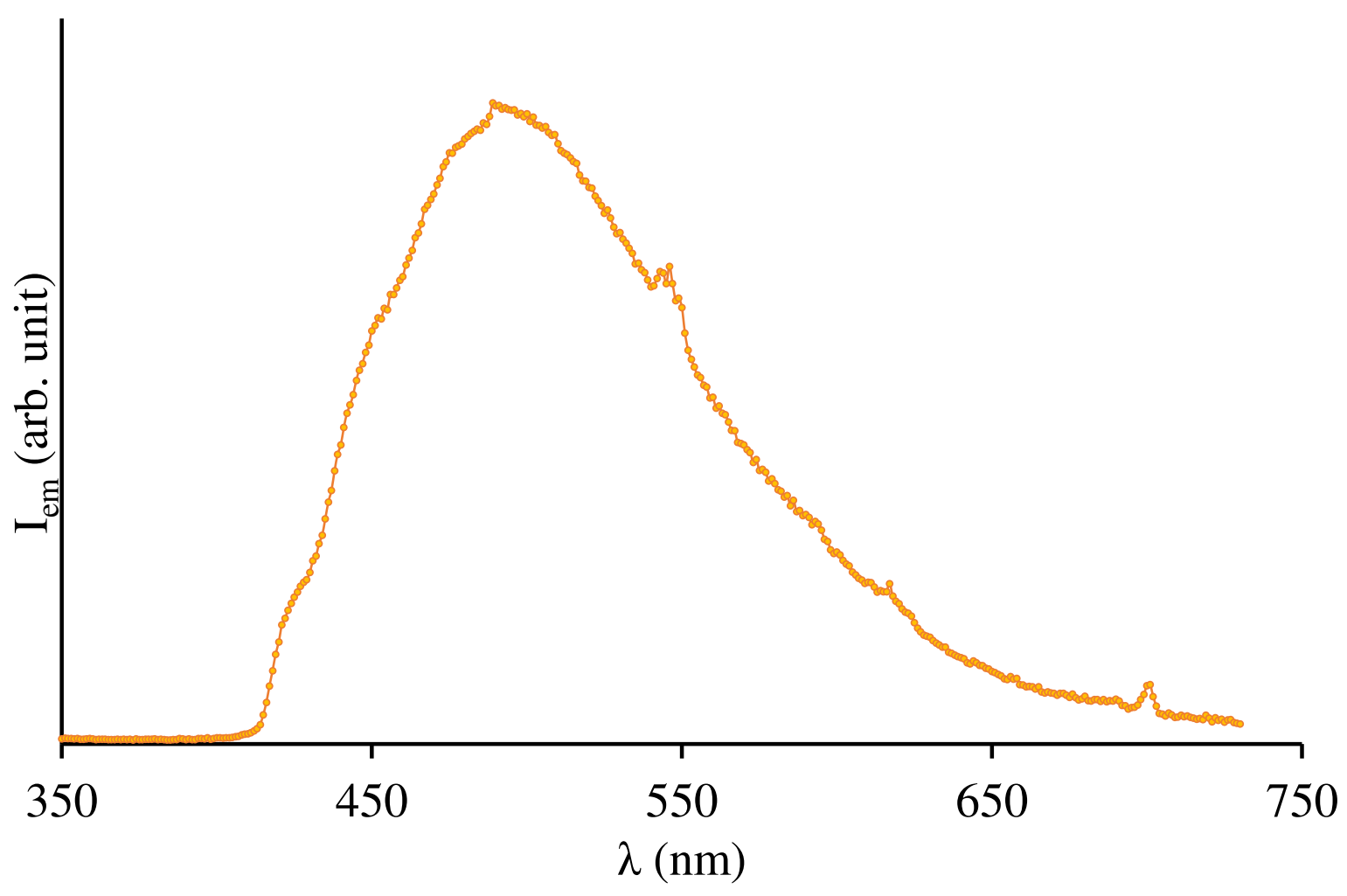

Fig. S11 | Emission spectra of $\left[\mathbf{G d}_{\mathbf{2}}\right]$ in the solid state at $77 \mathrm{~K}\left(\lambda_{\mathrm{ex}}=291 \mathrm{~nm}\right)$. The small intensity bands observed above $475 \mathrm{~nm}$ are most probably due to $\mathrm{Tb}$ (III) and $\mathrm{Eu}$ (III) contamination present in the $\mathrm{GdCl}_{3} \cdot 6 \mathrm{H}_{2} \mathrm{O}$ used to prepare the $\mathrm{Gd}$-complex. Efficient sensitization of $\mathrm{Eu}(\mathrm{III})$ and $\mathrm{Tb}(\mathrm{III})$ luminescence by 
4-picNO caused the appearance of $\mathrm{Tb}(\mathrm{III})$ - and $\mathrm{Eu}(\mathrm{III})$-based bands, despite their small concentrations ( 1\%).

S3.3.3. Analysis of ${ }^{7} F_{0} \rightarrow{ }^{5} D_{0}$ photoluminescence excitation structure
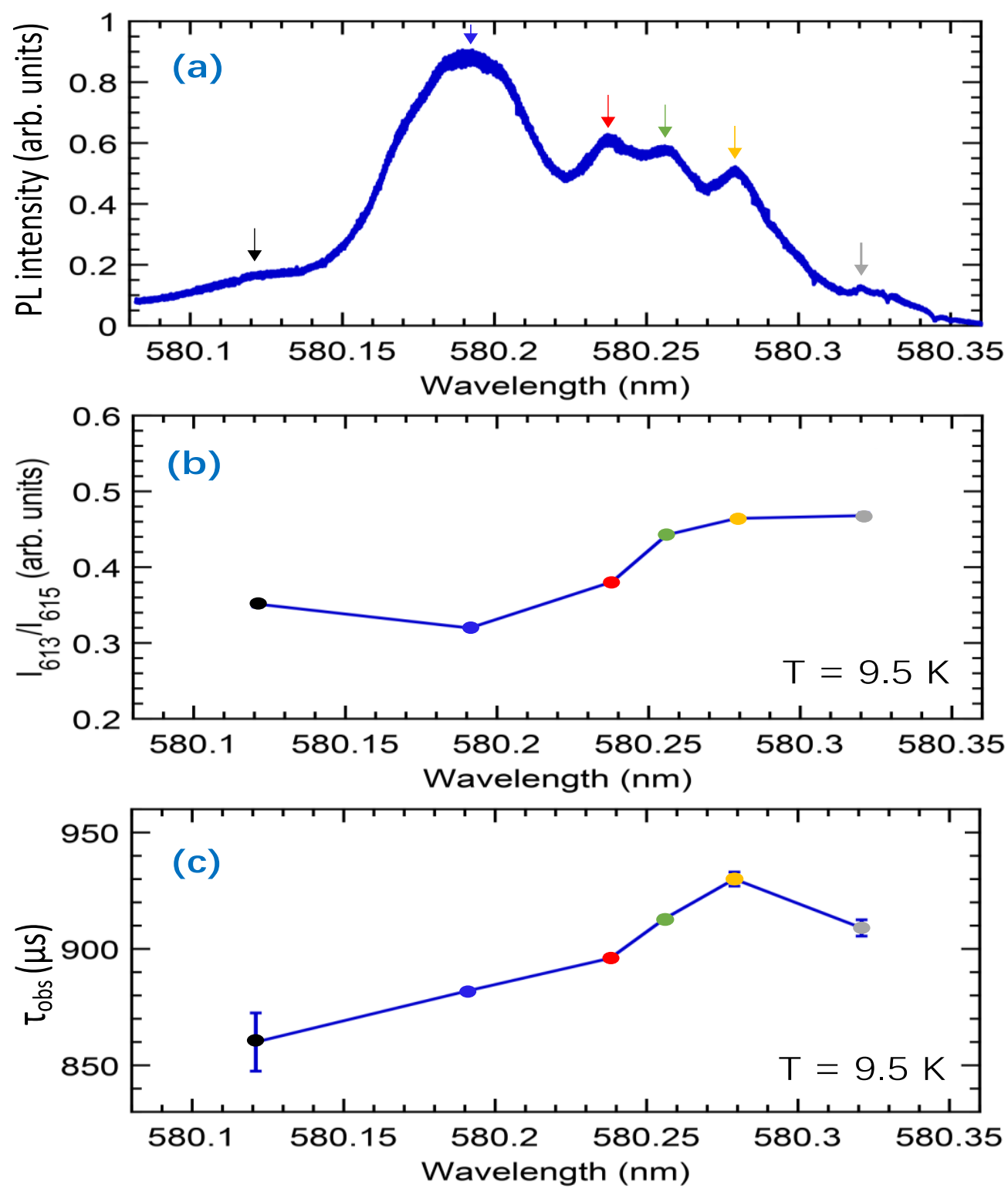

Fig. S12 | Low-temperature high-resolution PL spectroscopy. (a) Excitation spectrum for the Eu(III): ${ }^{7} \mathrm{~F}_{0} \rightarrow$ ${ }^{5} D_{0}$ transition obtained by monitoring the ${ }^{5} D_{0} \rightarrow{ }^{7} F_{J}(J=2-6) E u(I I I)$ emissions (see methods in main manuscript for further details). Colored arrows indicate the spectral positions at which we recorded emission spectra (b) and PL decays (c). (b) Intensity ratio between the $613 \mathrm{~nm}$ and the $615 \mathrm{~nm}$ emission peaks (associated to transitions from the ${ }^{5} \mathrm{D}_{0}$ singlet towards the ${ }^{7} \mathrm{~F}_{2}$ multiplet, see Fig. $2 \mathrm{~b}$ in the main script), as a function of ${ }^{7} \mathrm{~F}_{0} \rightarrow{ }^{5} \mathrm{D}_{0}$ excitation wavelength. The observed emission branching ratio variation indicates the selective excitation of different Eu(III) sub-sites. (c) Fluorescence lifetime as a function of ${ }^{7} \mathrm{~F}_{0}$ 
$\rightarrow{ }^{5} \mathrm{D}_{0}$ excitation wavelength. Lifetime values were obtained by single exponential fit to the ${ }^{5} \mathrm{D}_{0} \rightarrow{ }^{7} \mathrm{~F}_{\mathrm{J}}(\mathrm{J}=2$ 6 ) emissions decay curves (see methods in main manuscript for further details).

As shown and discussed in the main manuscript, the low temperature excitation spectrum of the ${ }^{7} \mathrm{~F}_{0} \rightarrow$ ${ }^{5} \mathrm{D}_{0} \mathrm{Eu}(\mathrm{III})$ transition show a main absorption peak at $580.185 \mathrm{~nm}$ (about $50 \mathrm{GHz}$ FWHM) surrounded by several partially resolved side peaks (Fig. S12(a)). To shed light on the origin of the side peaks, emission spectra and photoluminescence decay curves were recorded under selective laser excitation of the peaks. Clear differences were observed in emission peaks relative intensities (Fig. S12b) and fluorescence lifetimes (Fig. S12C), indicating that Eu(III) ions are found in different crystalline sub-sites. These sub-sites are most likely due to defects and/or minor configurational modifications in the Eu(III) local environment. The singly exponential decay curves obtained from all sub-sites indicate no energy transfer or interaction between them.

\section{S3.3.4. Photostability of $\left[\mathrm{Eu}_{2}\right]$}

Photostability of a molecular material is an important aspect determining the QIP utility of the material. Absence of photobleaching in the $\left[\mathrm{Eu}_{2}\right]$ complex was confirmed by monitoring the $\mathrm{Eu}(\mathrm{III})$ photoluminescence intensity under different excitation powers and different temperatures (Fig. S13). Furthermore, no signs of degradation of the photophysical properties of the complex were observed after repeated experiments on the same sample. This reflects the very stable charge state of Eu(III). ${ }^{4}$
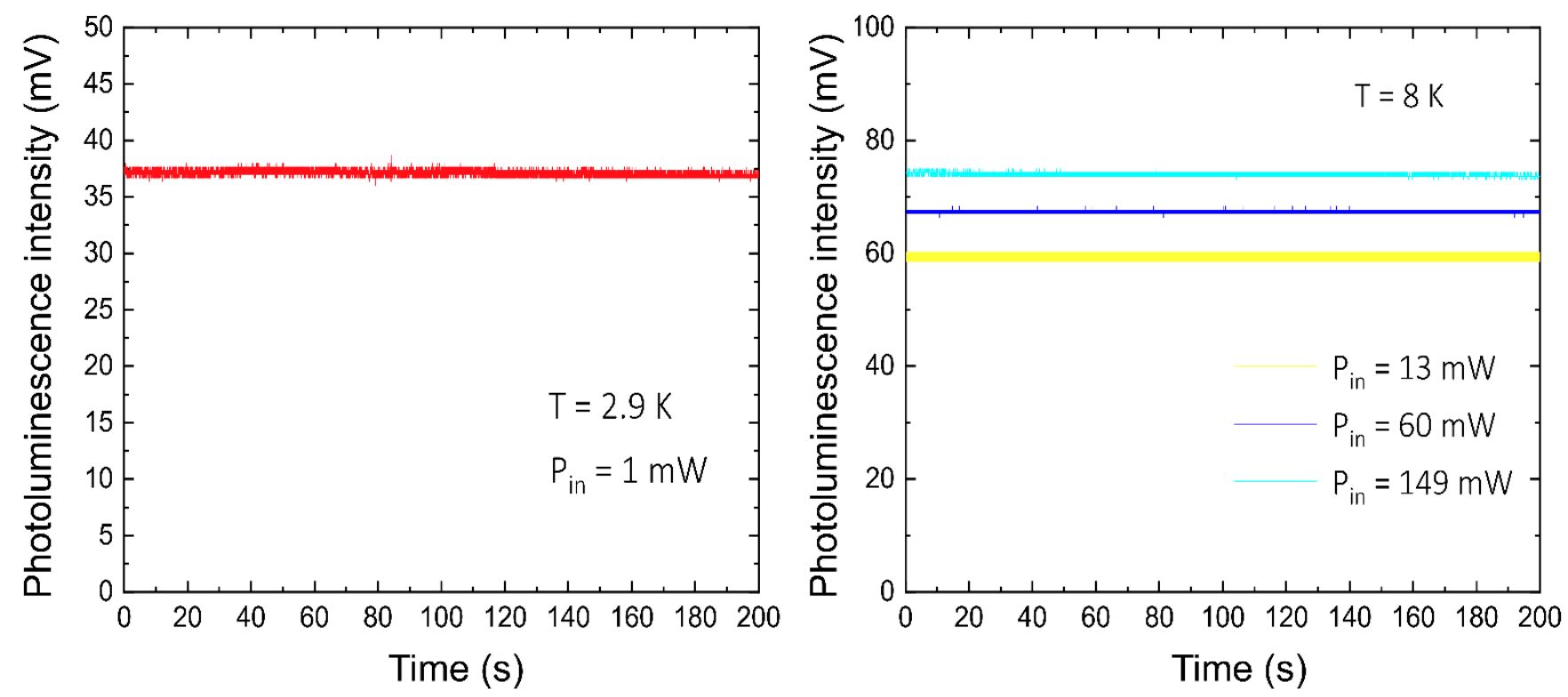

Fig. S13 | Photoluminescence intensity signal recorded over $200 \mathrm{~s}$ at different excitation powers and sample temperatures, showing the absence of photobleaching in $\left[\mathrm{Eu}_{2} \mathrm{Cl}_{6}(4-\mathrm{picNO})_{4}\left(\mu_{2}-4-\mathrm{picNO}\right)_{2}\right] \cdot 2 \mathrm{H}_{2} \mathrm{O}$. 


\section{S3.3.5. Homogeneous line broadening in $\left[\mathrm{Eu}_{2}\right]$ complex}

Processes that cause broadening of $\mathrm{Eu}(\mathrm{III})$ in [Eu 2 are mainly attributed to low-energy molecular vibrations or rotations through 2-phonon processes 5,6 . In addition, nuclear spins carried by $\mathrm{H}$ or $\mathrm{Cl}$ elements could also induce broadening, although the linewidths observed in $\mathrm{EuCl}_{3} \cdot 6 \mathrm{H}_{2} \mathrm{O}$ are on the order of a few $\mathrm{kHz}$. Spectral diffusion related to residual disorder caused by defects in the crystalline lattice (the so-called two-level systems, TLS) has also been observed in Eu(III) doped inorganic materials ${ }^{5}$ and studied in details in other systems such as single molecules embedded in organic crystals ${ }^{7}$. However, it should be noted that the narrow inhomogeneous linewidth observed in $\left[\mathrm{Eu}_{2}\right]$ does not point towards large disorder, but rather to a well crystallized system. In materials showing a comparable inhomogeneous linewidth,

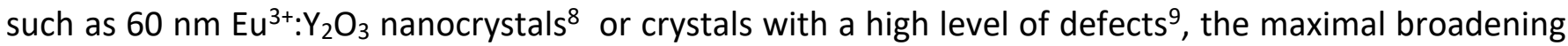
due to disorder is about $50 \mathrm{kHz} / \mathrm{K}$ and thus significantly lower than what we observe. Only in amorphous materials, such as glasses, can $\mathrm{Eu}(\mathrm{III})$ show broadenings of $10 \mathrm{~s}$ of $\mathrm{MHz} / \mathrm{K}^{10}$. Here, the inhomogeneous

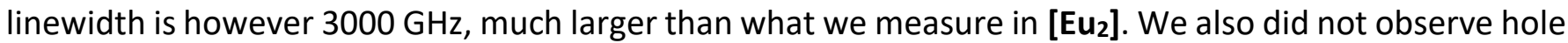
broadening as a function of the delay between burning and probing on a time scale up to $10 \mathrm{~s}$, which suggests that slow spectral diffusion processes do not dominate the homogeneous linewidth. These comparisons should however be taken with caution as the nature of defects, their dynamics and their

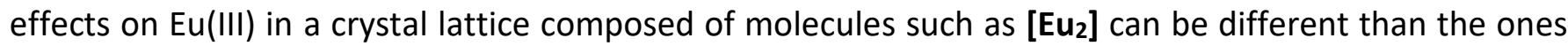
encountered in inorganic systems. Planar defects-such as grain boundaries, stacking faults, and step edges-cause defects in molecular lattices. For example, a layered arrangement of molecules in the bxc plane was observed in the crystal lattice of $\left[\mathrm{Eu}_{2}\right]$ (Fig. S5). A possible occurrence of step edges between

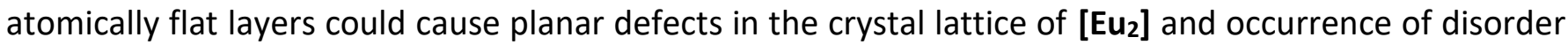
related spectral diffusion. Further studies such as measuring dependence of homogeneous linewidth on temperature would be needed to clarify the different contributions. Studies as a function of applied magnetic fields could also identify possible contributions from impurities or defects carrying electron spins, while Eu-Eu interactions could be probed by using mixed Y-Eu samples ${ }^{11}$.

Both lower temperature and high magnetic field could reduce Eu(III) homogeneous broadening and hence improve the quantum properties of $\left[\mathrm{Eu}_{2}\right]$ for applications in quantum technologies. However, current values do not prevent the coherent optical manipulation of spins using two-color pulses ${ }^{12}$. However, the fidelity with which spins can be controlled depends on the optical $\mathrm{T}_{2}$. As a figure of merit, one can compare it to the shortest optical pulse that can be applied to $\mathrm{Eu}(\mathrm{III})$ and which is limited by the hyperfine splittings. In ${ }^{153} \mathrm{Eu}$, these are typically in the order of $80 \mathrm{MHz}$, giving a minimal pulse duration $t$ of about $12.5 \mathrm{~ns}$ and a maximal fidelity $\exp \left(-\mathrm{t} / \mathrm{T}_{2}\right)=42 \%$. This is lower than what has been achieved in inorganic crystals $(96 \%)^{13}$. 
An improved molecular design and more favourable experimental conditions (lower temperature, high magnetic field) could increase $T_{2}$ and therefore achievable fidelities.

\section{S4. References}

1. Sheldrick, G. M. SHELXT - Integrated space-group and crystal-structure determination. Acta Crystallogr. Sect. Found. Adv. 71, 3-8 (2015).

2. Sheldrick, G. M. Crystal structure refinement with SHELXL. Acta Crystallogr. Sect. C Struct. Chem. 71, 3-8 (2015).

3. Thompson, L. C. \& Kuo, S. C. Structure and absorption and fluorescence spectra of $\mathrm{Eu}(\mathrm{PyNO})_{8}\left(\mathrm{C} 1 \mathrm{O}_{4}\right)_{3}$. J. Common Met. 148, 173-179 (1989).

4. Binnermans, K., Lanthanide-Based Luminescent Hybrid Materials, Chem. Rev. 109, 9, 4283-4374 (2009).

5. Goldner, P., Ferrier, A. \& Guillot-Noël, O. Rare Earth-Doped Crystals for Quantum Information Processing. in Handbook on the Physics and Chemistry of Rare Earths vol. 46 1-78 (Elsevier, 2015).

6. Riesen, H. \& Krausz, E. Persistent spectral hole-burning, luminescence line narrowing and selective excitation spectroscopy of the $R$ lines of $\mathrm{Cr}(\mathrm{III})$ tris(2,2'-bipyridine) in amorphous hosts. J. Chem. Phys. 97, 7902-7910 (1992).

7. Naumov, A. V. Low-temperature spectroscopy of organic molecules in solid matrices: from the Shpol'skii effect to laser luminescent spectromicroscopy for all effectively emitting single molecules, Phys.-Uspekhi $56,605-622$ (2013).

8. Perrot, A. et al. Narrow Optical Homogeneous Linewidths in Rare Earth Doped Nanocrystals. Phys. Rev. Lett. 111, (2013).

9. Flinn, G. P. et al. Anomalous optical dephasing in crystalline Y2O3: Eu3+. J. Lumin. 58, 374-379 (1994). 10. Macfarlane, R. M. \& Shelby, R. M. Homogeneous line broadening of optical transitions of ions and molecules in glasses. J. Lumin. 36, 179-207 (1987).

11. Kunkel, N. et al. Dephasing mechanisms of optical transitions in rare-earth-doped transparent ceramics. Phys. Rev. B 94, 184301 (2016).

12. Serrano, D., Karlsson, J., Fossati, A., Ferrier, A. \& Goldner, P. All-optical control of long-lived nuclear spins in rare-earth doped nanoparticles. Nat. Commun. 9, 2127 (2018).

13. Rippe, L., Julsgaard, B., Walther, A., Ying, Y. \& Kröll, S. Experimental quantum-state tomography of a solid-state qubit. Phys. Rev. A 77, 022307 (2008). 


\section{Figures}

(a)

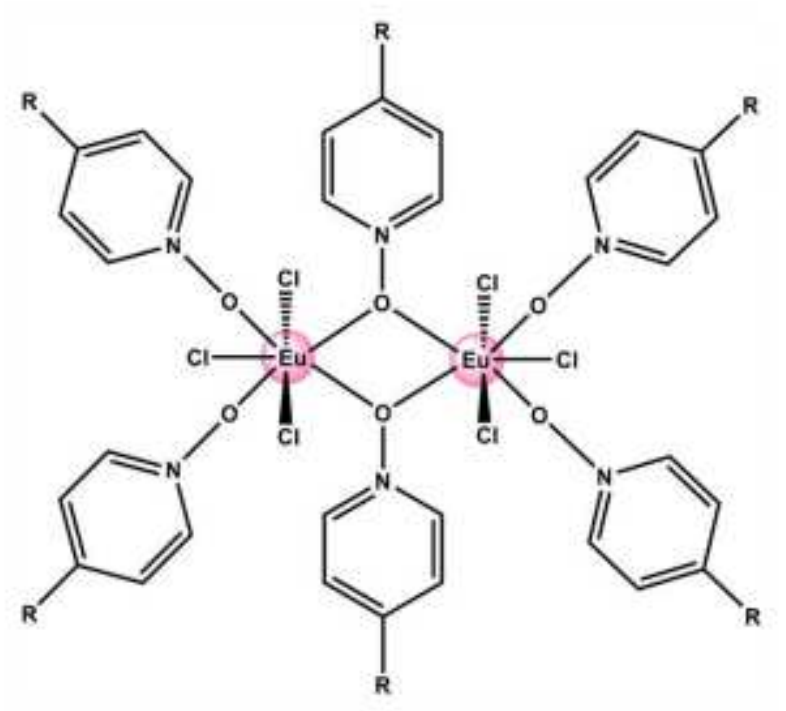

(b)

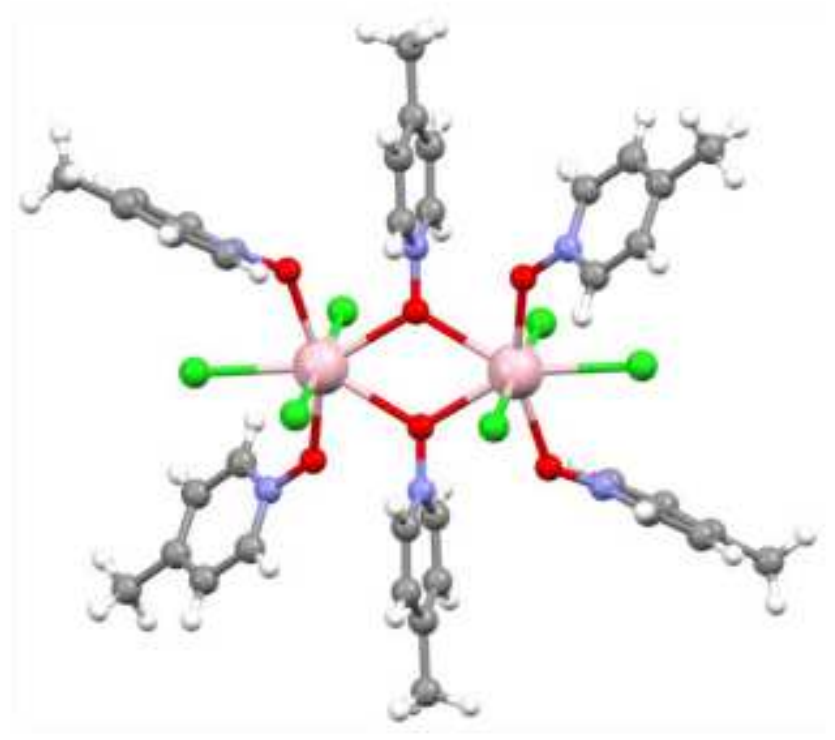

\section{Figure 1}

Structure of [Eu2Cl6(4-picNO)4( $\mu 2-4-p i c N O) 2] \cdot 2 \mathrm{H} 2 \mathrm{O}$. (a) Molecular structure of the complex showing the ligands $(\mathrm{R}=\mathrm{CH} 3)$ coordinating with the Eu(III) centers. The complex was prepared by treating EuCl3.6 20 with 4-picNO ligand dissolved in water followed by a recrystallization step from ethanol $(\mathrm{EtOH}) /$ ethyl acetate (EtOAc) solvent mixture. (b) X-ray crystal structure of the complex. Coordination geometry around each Eu(III) center of the complex is best described as pentagonal bipyramidal. The cocrystallized water molecules are omitted for clarity. Colour code: $\mathrm{C}$, grey; $\mathrm{Cl}$, green; Eu, pink; $\mathrm{H}$, white; $\mathrm{N}$, blue; 0 , red. 
(a)

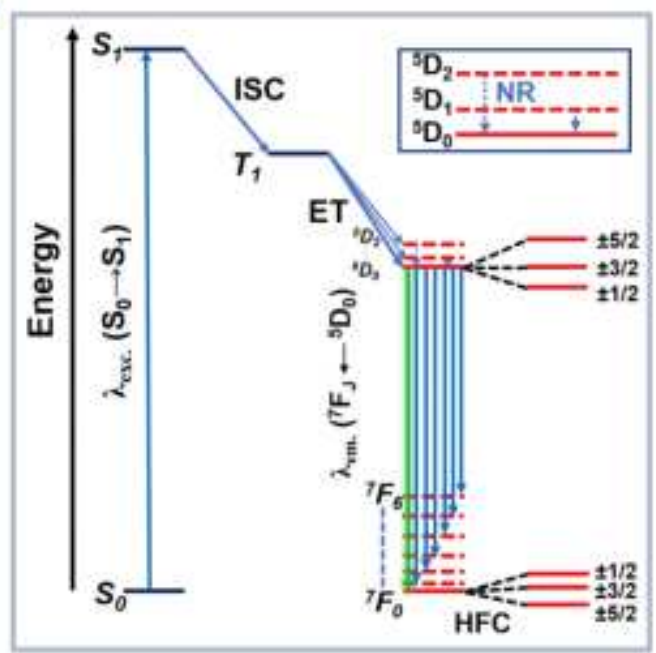

(c)

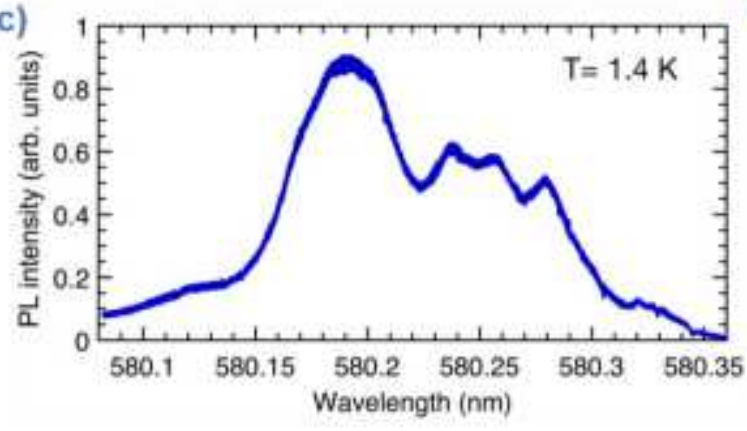

(b)

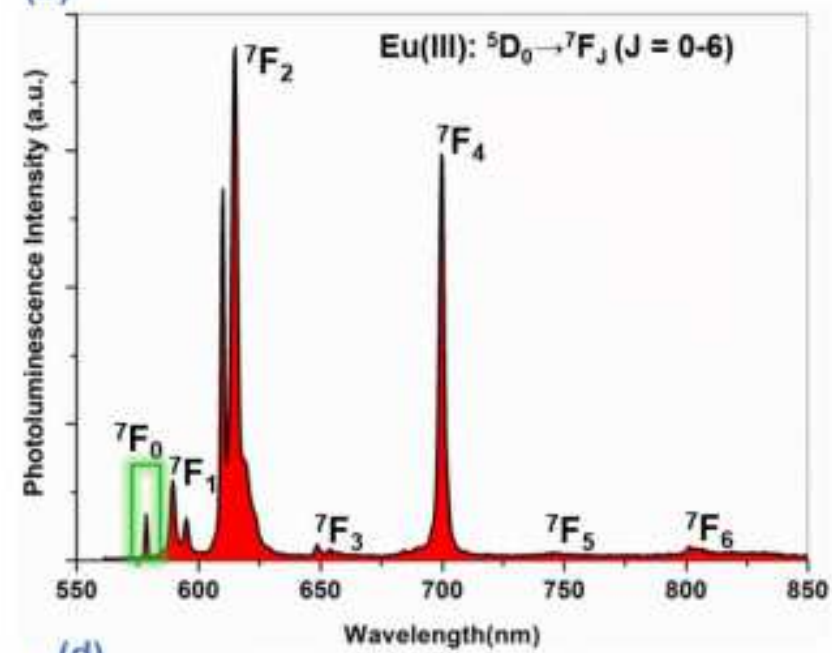

(d)

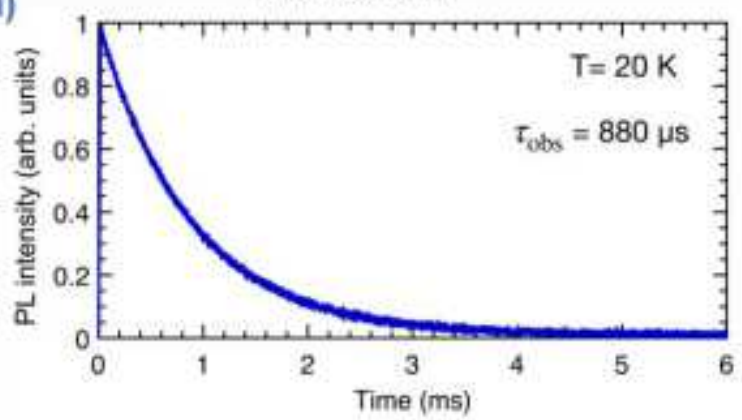

Figure 2

Photophysical properties of [Eu2] in the solid-state. (a) Mechanism of PL sensitization in Eu(III) complexes: The Eu(III)-based 5DJ ( $\mathrm{J}=0-2)$ receiving levels are populated after a series of excitation, intersystem crossing (ISC), and T1区5DJ energy transfer (ET) processes. The co-populated excited 5D2 and 5D1 levels non-radiatively (NR) relax to 5D0 level (inset);radiative relaxation of 5D0 level to ground $7 F J(\mathrm{~J}=0-6)$ crystal field levels manifests as line-like luminescence. The 5D0冈7F0 transition is suited for QIP applications due to its narrow linewidth and long coherence lifetimes of the nuclear spin states$\pm 5 / 2, \pm 3 / 2, \pm 1 / 2$ ( I = 5/2 for 151Eu/153Eu)-associated with the 7F0 ground-state level. (b) Photoluminescence spectrum showing the 5D0囚7FJ $(\mathrm{J}=0-6)$ transitions in the visible and near-IR range $(\lambda$ exc. $=330 \mathrm{~nm}) .(\mathrm{c})$ Photoluminescence excitation (PLE) spectrum of the 5D087F0 transition of [Eu2] measured at $1.4 \mathrm{~K}$. An inhomogeneous linewidth (Гinh) of $50 \mathrm{GHz}$ is calculated for the main peak centered at $580.185 \mathrm{~nm}$ (vac.) (d) Luminescence decay of the 5D0 excited-state of [Eu2] measured at 20 $\mathrm{K}$ under resonant excitation at $580.185 \mathrm{~nm}$. An excited-state lifetime (tobs) of 880 us was obtained from a single exponential fit of the decay. 

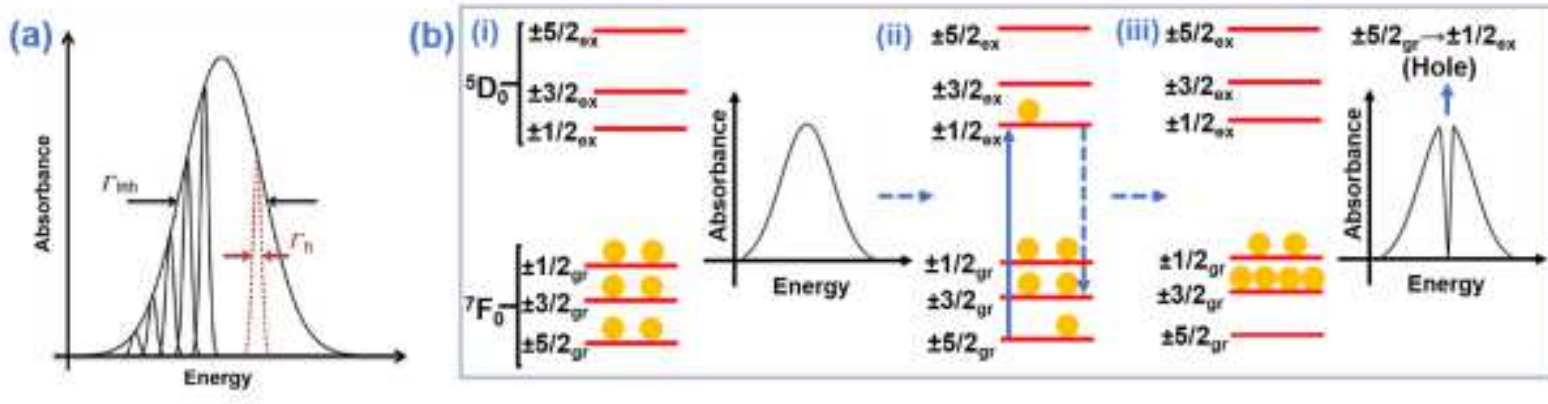

(c)

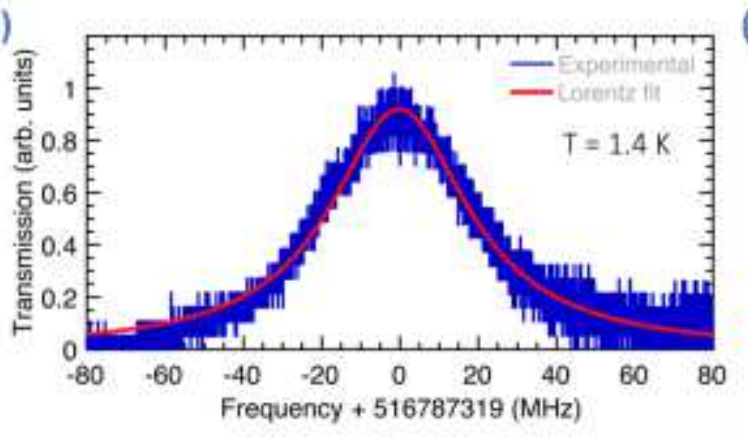

(d)

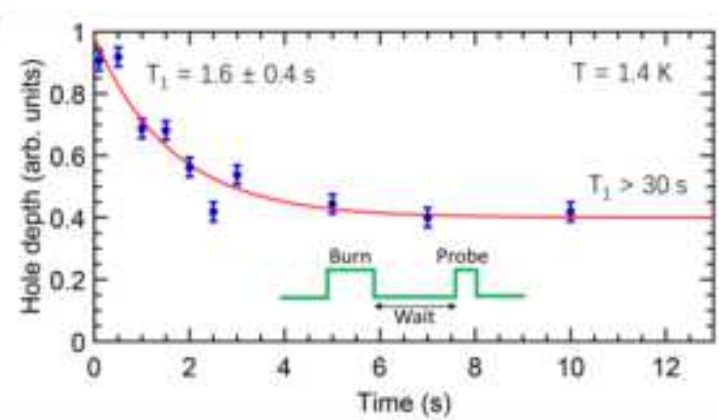

\section{Figure 3}

Spectral hole burning (SHB) in the 5D0ه7F0 transition of [Eu2]. (a) An inhomogeneously ( $\Gamma$ inh) broadened line is composed of narrow homogeneously $(\Gamma \mathrm{h})$ broadened lines. Selective optical excitation of one or several homogeneously broadened lines is used to burn spectral holes with implications for QIP applications. (b) Simplified mechanism of SHB. In the diagram, $\pm 1 / 2 \mathrm{gr}, \pm 3 / 2 \mathrm{gr}, \pm 5 / 2 \mathrm{gr}$ and $\pm 1 / 2 \mathrm{ex}$, $\pm 3 / 2 \mathrm{ex}, \pm 5 / 2 \mathrm{ex}$ correspond to nuclear spin levels associated with the ground 7F0 and excited 5D0 states, respectively. (i) A laser scan reveals an inhomogeneously broadened absorption spectrum, like the one shown in (a), due to the excitation of multiple ions ensembles with infinitesimally small energy difference. (ii) Selective and continuous laser pumping transfers population from one of the ground-state spin levels to an excited-state spin level. This is illustrated for ions with the $\pm 5 / 2 \mathrm{gr}$ ( $7 F 0$ ) to $\pm 1 / 2 \mathrm{ex}$ ( $5 \mathrm{D0}$ ) transition resonant with the laser. (iii) Population relaxation from level $\pm 1 / 2$ ex to level $\pm 3 / 2 \mathrm{gr}$, under the ambit of level $\pm 3 / 2$ gr having sufficiently longer lifetime than levels $\pm 1 / 2 \mathrm{gr}$ and $\pm 5 / 2 \mathrm{gr}$, results in a decreased population in level $\pm 5 / 2 \mathrm{gr}$. Since the spin level splittings are much lower than the optical inhomogeneous broadening, the laser will excite other ions along transitions from the $\pm 3 / 2 \mathrm{gr}$ or $\pm 1 / 2 \mathrm{gr}$ levels, which will therefore be emptied. This finally results in a hole in the inhomogeneously broadened absorption spectrum. (c) Spectral hole burned in the 5D0®7F0 transition of [Eu2]. A Lorentzian fit reveals an FWHM of $43 \pm 2 \mathrm{MHz}$, corresponding to $\Gamma \mathrm{h}=22 \pm 1 \mathrm{MHz}$. A T2opt $=\sim 14.5 \mathrm{~ns}$ is calculated using the relation $\Gamma \mathrm{h}$ $=(1 / \pi T 2 o p t)$. (d) The decay of the hole depth as a function of delay time before readout, showing the relaxation rate of the nuclear spin levels in the binuclear complex. Figures (a) and (b) are partially reproduced with permission from reference 23 . 